TRANSACTIONS OF THE

AMERICAN MATHEMATICAL SOCIETY

Volume 350, Number 12, December 1998, Pages 5047-5066

S $0002-9947(98) 02025-\mathrm{X}$

\title{
CHARACTER SUMS ASSOCIATED TO FINITE COXETER GROUPS
}

\author{
JAN DENEF AND FRANÇOIS LOESER
}

\begin{abstract}
The main result of this paper is a character sum identity for Coxeter arrangements over finite fields which is an analogue of Macdonald's conjecture proved by Opdam.
\end{abstract}

\section{INTRODUCTION}

Throughout this paper $\mathbf{F}$ will denote a finite field of characteristic $p$ different from 2.

We first slightly reformulate Macdonald's conjecture in a form which has a direct analogue over $\mathbf{F}$. Let $G$ be a finite subgroup of $\mathrm{GL}_{n}(\mathbf{R})$ generated by reflections and let $q$ be a positive definite quadratic form which is invariant under $G$. Let $\mathcal{A}_{G}$ be the associated arrangement consisting of the reflection hyperplanes. Let $\ell_{1}, \ldots, \ell_{N}$ be equations for the $N$ different reflection hyperplanes. Set $\Delta(x)=\left(\prod_{i=1}^{N} \ell_{i}\right)^{2}$ and let $d_{1}, \ldots, d_{n}$ be the degrees of $G$. Macdonald's conjecture [19] is the following equality,

$$
\int_{\mathbf{R}^{n}} \Delta(x)^{s} e^{-\left(\sum_{i} x_{i}^{2}\right) / 2} d x=(2 \pi)^{n / 2} \prod_{i=1}^{n} \frac{\Gamma\left(d_{i} s+1\right)}{\Gamma(s+1)},
$$

when $q(x)=\sum_{i} x_{i}^{2}$ and the $\ell_{i}$ are normalized in such a way that $\left\|\ell_{i}\right\|=\sqrt{2}$. If we drop the condition that the $\ell_{i}$ are normalized, and if we replace $x$ by $\sqrt{2} x$, we obtain

$$
\int_{\mathbf{R}^{n}} \Delta(x)^{s} e^{-\sum_{i} x_{i}^{2}} d x=\pi^{n / 2}\left(\prod_{i=1}^{N} \frac{\left\|\ell_{i}\right\|^{2}}{4}\right)^{s} \prod_{i=1}^{n} \frac{\Gamma\left(d_{i} s+1\right)}{\Gamma(s+1)} .
$$

Hence for $q$ an arbitrary positive definite quadratic form which is invariant under $G$, we obtain

$$
\int_{\mathbf{R}^{n}} \Delta(x)^{s} e^{-q(x)} d x=\pi^{n / 2} \kappa^{s}\left(\prod_{i=1}^{n} \frac{\Gamma\left(d_{i} s+1\right)}{\Gamma(s+1)}\right)(\operatorname{discr} q)^{-\frac{1}{2}},
$$

with

$$
\kappa=\prod_{i=1}^{N} \frac{q\left(\ell_{i}\right)}{4}
$$

Received by the editors March 29, 1996 and, in revised form, January 24, 1997.

1991 Mathematics Subject Classification. Primary 11T24, 11L05; Secondary 33C80.

Key words and phrases. Character sums, finite fields, Coxeter groups, monodromy, $\ell$-adic cohomology. 
where we consider $\ell_{i}$ in $q\left(\ell_{i}\right)$ as a vector in $\mathbf{R}^{n}$, identifying $\mathbf{R}^{n}$ with its dual, by means of the quadratic form $q$. Observe that

$$
\sqrt{q\left(\ell_{i}\right)}=\operatorname{Max}_{\substack{x \in \mathbf{R}^{n} \\ q(x)=1}} \ell_{i}(x) .
$$

Formula (0.1) can be reformulated as

$$
\int_{K} \Delta(x)^{s} e^{-q(x)} \sqrt{\Delta(x)} d x=\pi^{n / 2} \kappa^{s+\frac{1}{2}}\left(\prod_{i=1}^{n} \frac{\Gamma\left(d_{i}\left(s+\frac{1}{2}\right)\right)}{\Gamma\left(s+\frac{1}{2}\right)}\right)(\operatorname{discr} q)^{-\frac{1}{2}},
$$

where $K=\mathbf{R}^{n} / G$. Observe that $\sqrt{\Delta(x)} d x$ is a $G$-invariant differential form.

Over $\mathbf{F}$, there is the notion of a Coxeter arrangement (1.4). It is a triple, $A=(V, G, q)$, where $V$ is a finite-dimensional $\mathbf{F}$-vector space, $G$ a finite subgroup of GL $(V)$ generated by reflections, and $q$ a $G$-invariant nondegenerate symmetric bilinear form on $V$. If $p$ does not divide $|G|$, one may define the degrees of $G$, $d_{1}, \ldots, d_{n}(1.5)$. Let $\ell_{1}, \ldots, \ell_{N}$ be equations for the $N$ different reflection hyperplanes. Put $\Delta(x)=\left(\prod_{i=1}^{N} \ell_{i}\right)^{2}$. Because $p \neq 2$, we may define an element $\kappa$ of $\mathbf{F}$ by (0.2). Fix a nontrivial additive character $\psi: \mathbf{F} \rightarrow \mathbf{C}$. The analogue of the integral in (0.4) will be the character sum

$$
S_{G}(\chi):=\sum_{x \in(U / G)(\mathbf{F})} \chi(\Delta(x)) \psi(q(x)),
$$

where $\chi: \mathbf{F}^{\times} \rightarrow \mathbf{C}^{\times}$is a multiplicative character and $U$ denotes the complement of the hypersurface $\Delta(x)=0$ in $V$. (We write $q(x)$ for the quadratic form $q(x, x)$ associated to the bilinear form $q$, and use the standard notation $(U / G)(\mathbf{F})$ to denote the set of F-rational points on the quotient space $U / G$.) The analogue of the Gamma function will be the Gauss sum $-g(\chi)$, where $g(\chi):=-\sum_{x \in \mathbf{F} \times} \chi(x) \psi(x)$. Our main result is the following.

Main Theorem. Let $A=(V, G, q)$ be a Coxeter arrangement over $\mathbf{F}$. Assume that $p$ does not divide $|G|$. Then $\kappa \neq 0$ and, for every multiplicative character $\chi: \mathbf{F}^{\times} \rightarrow \mathbf{C}^{\times}$,

$$
S_{G}(\chi)=(-1)^{n} \phi(\operatorname{discr} q) g(\phi)^{n} \phi(\kappa) \chi(\kappa) \prod_{i=1}^{n} \frac{g\left((\phi \chi)^{d_{i}}\right)}{g(\phi \chi)},
$$

where $\phi$ denotes the unique multiplicative character of $\mathbf{F}$ of order 2.

Note the analogy with Macdonald's formula (0.4), replacing $\sqrt{\pi}=\Gamma(1 / 2)$ by $-g(\phi)$. (For some more remarks on this analogy, see (4.6) below.) Our proof of the Main Theorem depends on Macdonald's formula (0.1) at one single point, namely for the proof of Theorem 3.3 we need to know that, with the notation of $(0.1)$,

$$
\operatorname{Max}\left\{\Delta(x) \mid x \in \mathbf{R}^{n}, q(x)=1\right\}=\kappa N^{-N} \prod_{i=1}^{n} d_{i}^{d_{i}},
$$

which follows directly from (0.1), by elementary calculus. Without relying on Macdonald's formula we can nevertheless prove the Main Theorem for some $\kappa$ in $\mathbf{F} \backslash\{0\}$ which is independent of $\chi$ without the assertion that $\kappa$ is given by $(0.2)$.

In the special case where $G$ is the symmetric group $\mathcal{S}_{n}$, the Main Theorem has been proved for all $p \neq 2$ by R. Evans [13], extending the results of G. Anderson on Selberg sums [1]. For the history and applications of such sums, we refer to 
[11] and [12]. For recent work on character sums related to relative invariants of prehomogeneous vector spaces, see [6].

Our proof of the Main Theorem is entirely based on the cohomological interpretation of character sums, using the Grothendieck-Lefschetz trace formula (see [26] Sommes trig. and [15]). In the present situation, the cohomology is concentrated in middle dimension (Proposition 2.1.2) and has rank 1 (Corollary 4.3.2 and formula (4.4.6)), so that we have only to calculate the determinant of the Frobenius action on the cohomology, which is done by Laumon's product formula [16] 3.2.1.1.

The plan of the paper is the following. In the first section we study Coxeter arrangements over finite fields and their liftings to characteristic zero. In section 2 we study character sums associated to central arrangements that are invariant under a finite group. This will allow us to evaluate $S_{G}(\phi)$. In the next section we review results on monodromy and critical values for Coxeter arrangements from [10] and [7]. In the last section, using all the previous results, we prove the Main Theorem.

Most of the present work dates from 1992. It has been presented, under some additional hypotheses, in lectures by the first named author in Orsay (SAGA) in November 1992 and in Amsterdam in January 1993.

\section{Liftable Coxeter arrangements over $\mathbf{F}$}

1.1. Let $V$ be a finite dimensional vector space over a field. By a hyperplane arrangement in $V$ we mean a finite set of affine hyperplanes in $V$. If all the hyperplanes contain 0 , the arrangement is said to be central. We call an endomorphism of $V$ a reflection if it has order 2 and fixes pointwise some hyperplane.

We define a classical Coxeter arrangement as a triple $A=(V, G, q)$, where $V$ is a finite dimensional vector space over $\mathbf{R}, G$ is a finite subgroup of GL $(V)$ generated by reflections, and $q$ is a $G$-invariant positive definite symmetric bilinear form on $V$. We define a Coxeter arrangement over $\mathbf{C}$ as a triple $A=(V, G, q)$, where $V$ is a finite dimensional vector space over $\mathbf{C}, G$ is a finite subgroup of $\mathrm{GL}(V)$ generated by reflections, and $q$ is a $G$-invariant nondegenerate symmetric bilinear form on $V$, which arises by extension of scalars from a classical Coxeter arrangement. (In fact, by the argument given at the end of the proof of Proposition 1.6, the last condition in this definition is automatically verified.)

Similarly, when $T$ is a subring of $\mathbf{C}$ with fraction field $K$, a Coxeter arrangement over $T$ will be a triple $A=(M, G, q)$, where $M$ is a free $T$-module of finite rank, $G$ a finite subgroup of $\mathrm{GL}(M)$ generated by reflections, and $q$ a $G$-invariant nondegenerate symmetric bilinear form on $M$, which induces by extension of scalars a Coxeter arrangement over $\mathbf{C}$.

The finite set of reflection hyperplanes in $M \otimes_{T} K$ defines a central hyperplane arrangement in $M \otimes_{T} K$ which we denote by $\mathcal{A}_{G}$.

1.2. Let $T$ be a discrete valuation ring with fraction field $K$. Let $\mathfrak{P}$ be the maximal ideal of $T$ with residue field $\bar{K}=T / \mathfrak{P}$. Let $M$ be a free $T$-module of finite rank, and set $V=M \otimes_{T} K, \bar{V}=M \otimes_{T} \bar{K}$. We denote the reduction map $M \rightarrow \bar{V}$ by $x \mapsto \bar{x}$. For any linear subspace $W$ in $V$, we denote by $\bar{W}$ the reduction modulo $\mathfrak{P}$ of $W$ which is defined by

$$
\bar{W}=\{\bar{x} \mid x \in W \cap M\} .
$$

Observe that $\operatorname{dim}_{K} W=\operatorname{dim}_{\bar{K}} \bar{W}$. 
Definition 1.2.1. Let $\mathcal{A}=\left(H_{i}\right)_{i \in J}$ be a central arrangement in $V$. We say that $\mathcal{A}$ has good reduction mod $\mathfrak{P}$ if, for any $I \subset J$,

$$
\overline{\bigcap_{i \in I} H_{i}}=\bigcap_{i \in I} \overline{H_{i}} .
$$

For $\mathcal{A}$ an arrangement in $V$ consider the arrangement $\overline{\mathcal{A}}$ consisting of the hyperplanes $\bar{H}$ with $H$ in $\mathcal{A}$. Denote the lattices of $\mathcal{A}$ and $\overline{\mathcal{A}}$ by $L(\mathcal{A})$ and $L(\overline{\mathcal{A}})$. There is a canonical inclusion preserving map

$$
\theta:\left\{\begin{array}{l}
L(\mathcal{A}) \underset{ }{\longrightarrow} L(\overline{\mathcal{A}}) \\
X \mapsto \bigcap_{H \supset \mathcal{A}} \bar{H} .
\end{array}\right.
$$

Proposition 1.2.2. If $\mathcal{A}$ has good reduction mod $\mathfrak{P}$, then $\theta$ is an isomorphism of lattices.

Proof. This is straightforward because $\theta(X)=\bar{X}$ when $\mathcal{A}$ has good reduction. Also the equality $\operatorname{dim}_{K} W=\operatorname{dim}_{\bar{K}} \bar{W}$ holds for any linear subspace $W$ of $V$.

1.3. We assume the notation of (1.2). Suppose that $T \subset \mathbf{C}$ and let $(M, G, q)$ be a Coxeter arrangement over $T$. Thus, in particular, $G \subset \mathrm{GL}(M)$. Let $\mathcal{A}_{G}$ be the central arrangement in $V$ consisting of all the reflection hyperplanes of $G$. We denote the image of $G$ in $\operatorname{GL}(\bar{V})$ by $\bar{G}$ and we choose linear forms $\ell_{i}$ over $T$ which define the hyperplanes of $\mathcal{A}_{G}$ and which are not zero modulo $\mathfrak{P}$. Let $\kappa$ be as in (0.2).

Proposition 1.3.1. Assume that $\mathfrak{P}$ does not contain $|G|$. Then $\mathcal{A}_{G}$ has good reduction mod $\mathfrak{P}$. Furthermore the canonical morphism $G \rightarrow \bar{G}$ is an isomorphism, and $G$ acts freely on the complement in $\bar{V}$ of the hyperplanes in $\overline{\mathcal{A}_{G}}$.

Proof. For the first statement it suffices to show that for all $I \subset J$

$$
\operatorname{dim} \bigcap_{i \in I} \overline{H_{i}} \leq \operatorname{dim} \overline{\bigcap_{i \in I} H_{i}} .
$$

We may suppose that the $H_{i}$ 's with $i$ in $I$ are linearly independent. Let $s_{i}$ be the reflection with respect to $H_{i}$ and set $\gamma=\prod_{i \in I} s_{i}, \operatorname{Fix}(\gamma)=\{x \in V \mid \gamma(x)=x\}$ and $\operatorname{Fix}(\bar{\gamma})=\{\bar{x} \in \bar{V} \mid \bar{\gamma}(\bar{x})=\bar{x}\}$. From the proof of Theorem 6.27 (2) on p.225 of [22] we see that $\operatorname{Fix}(\gamma)=\bigcap_{i \in I} H_{i}$. Since $\bar{H}_{i} \subset \operatorname{Fix}\left(\bar{s}_{i}\right), \operatorname{dim} \bigcap_{i \in I} \overline{H_{i}} \leq \operatorname{dim} \operatorname{Fix}(\bar{\gamma})$. But this last dimension is equal to the number of eigenvalues of $\bar{\gamma}$ which are equal to 1 , i.e., the number of eigenvalues of $\gamma$ which are congruent to $1 \bmod \mathfrak{P}$ (counting multiplicities and enlarging $T$ so that it contains all eigenvalues of $\gamma$ ). But if $\lambda$ is an eigenvalue of $\gamma$ which is congruent to $1 \bmod \mathfrak{P}$, then $\lambda=1$, because $\lambda^{|G|}=1$, and $\mathfrak{P}$ does not divide $|G|$. But the number of eigenvalues of $\gamma$ which are equal to 1 (counting multiplicities) is equal to $\operatorname{dim} \operatorname{Fix}(\gamma)$. We now deduce the first statement from the equalities

$$
\operatorname{dim} \operatorname{Fix}(\gamma)=\operatorname{dim} \bigcap_{i \in I} H_{i}=\operatorname{dim} \overline{\bigcap_{i \in I} H_{i}} .
$$

For the second statement we just have to observe that if an element $\gamma$ of $G$ is in the kernel of the morphism $G \rightarrow \bar{G}$, all the eigenvalues of $\gamma$ are congruent to 1 mod $\mathfrak{P}$, hence are equal to 1 by the above argument. The order of $\gamma$ being finite, we conclude that $\gamma$ is equal to 1 . Finally the third assertion follows from the well known fact ( $c f$. [4] Ch.V, $\S 3$ Proposition 3) that $G$ acts freely on the complement 
in $V$ of the hyperplanes in $\mathcal{A}_{G}$, and from the observation that $\operatorname{Fix}(\bar{\alpha})=\overline{\operatorname{Fix}(\alpha)}$, for all $\alpha$ in $G$.

For each reflection $s \in G$ we choose an eigenvector $\alpha_{s} \in M$ of $s$ which is orthogonal (with respect to $q$ ) to the reflection hyperplane of $s$.

Set $\Phi=\left\{\alpha_{s} \mid s\right.$ reflection in $\left.G\right\}$. Note that

$$
s(x)=x-2 \frac{q\left(x, \alpha_{s}\right)}{q\left(\alpha_{s}, \alpha_{s}\right)} \alpha_{s} \quad \text { for all } x \in V .
$$

If $q(\alpha, \alpha) \notin \mathfrak{P}$ for each $\alpha \in \Phi$, then,

$$
\bar{s}(\bar{x})=\bar{x}-2 \frac{\bar{q}\left(\bar{x}, \bar{\alpha}_{s}\right)}{\bar{q}\left(\bar{\alpha}_{s}, \bar{\alpha}_{s}\right)} \bar{\alpha}_{s} \quad \text { for all } \bar{x} \in \bar{V} .
$$

Denote by $\bar{q}$ the symmetric bilinear form induced by $q$ on $\bar{V}$.

Lemma 1.3.2. Assume that $G$ is essential (meaning that $V^{G}=\{0\}$ ), that $\mathfrak{P}$ does not contain $|G|$, and that $q(\alpha, \alpha) \notin \mathfrak{P}$ for all $\alpha \in \Phi$. Then the bilinear form $\bar{q}$ is nondegenerate.

Proof. Assume that there exists $\bar{x} \in \bar{V}$ with $\bar{q}(\bar{x}, \bar{y})=\overline{0}$ for all $\bar{y} \in \bar{V}$. Then (2) implies that $\bar{s}(\bar{x})=\bar{x}$ for every reflection $s$ in $G$. Let $H_{s}$ be the reflection hyperplane of the reflection $s$. Then $\bar{x} \in \bar{H}_{s}$ because otherwise $\bar{s}$ is the identity and all eigenvalues of $s$ would be congruent to $1 \bmod \mathfrak{P}$. Hence $\bar{x} \in \bigcap_{H \in \mathcal{A}_{G}} \bar{H}=$ $\overline{\bigcap_{H \in \mathcal{A}_{G}} H}=\overline{V^{G}}=\overline{0}$ by Proposition 1.3.1.

Proposition 1.3.3. Assume that $\mathfrak{P}$ does not contain $|G|$ and that the bilinear form $\bar{q}$ is nondegenerate. Then $\kappa \in T \backslash \mathfrak{P}$, and, for any $I \subset J$, the restriction of $\bar{q}$ to $\bigcap_{i \in I} \bar{H}_{i}$ is nondegenerate.

Proof. Observe first that the characteristic of $\bar{K}$ is not 2 because 2||$G \mid$. For each reflection $s \in G$, choose an eigenvector $\alpha_{s} \in M$ of $s$ which is orthogonal (with respect to $q$ ) to the reflection hyperplane of $s$ such that $\bar{\alpha}_{s} \neq 0$. Since $\bar{q}$ is nondegenerate there exists $x \in M$ such that $\bar{q}\left(\bar{x}, \bar{\alpha}_{s}\right) \neq 0$. Thus $q\left(x, \alpha_{s}\right) \not \equiv 0 \bmod \mathfrak{P}$. Because $s(x) \in M$, formula $(1)$ yields $q\left(\alpha_{s}, \alpha_{s}\right) \not \equiv 0 \bmod \mathfrak{P}$. This implies that $\kappa \in T \backslash \mathfrak{P}$. Set $U=\bigcap_{i \in I} H_{i}$, thus $\bar{U}=\bigcap_{i \in I} \bar{H}_{i}$. We have to show that the restriction of $\bar{q}$ to $\bar{U}$ is nondegenerate. Because $\bar{q}$ is nondegenerate, it suffices to prove that the restriction to $(\bar{U})^{\perp}$ is nondegenerate. We have $\overline{\left(U^{\perp}\right)}=(\bar{U})^{\perp}$ because, clearly, $\overline{\left(U^{\perp}\right)} \subset(\bar{U})^{\perp}$ and both have the same dimension $n-\operatorname{dim} U$. Thus it remains to prove that $\bar{q}$ is nondegenerate on $\overline{\left(U^{\perp}\right)}$. Let $W$ be the subgroup of $G$ which is generated by the reflections with respect to the reflection hyperplanes of $G$ which contain $U$. Clearly, $W$ is a finite Coxeter group in $U^{\perp}$ and $W \subset \mathrm{GL}\left(U^{\perp} \cap M\right)$. It is easy to see that the hypothesis of Lemma 1.3.2 is satisfied if we replace $V$ by $U^{\perp}, M$ by $U^{\perp} \cap M$ and $q$ by its restriction to $U^{\perp} \cap M$. Indeed $\left(U^{\perp}\right)^{W}=\{0\}$ because $V^{W}$ is contained in $U$, and thus $\left(U^{\perp}\right)^{W} \subset U^{\perp} \cap U=\{0\}$ since $q_{\mid U}$ arises from a positive definite form over R. Hence Lemma 1.3.2 shows that $\overline{\left(q_{\mid U^{\perp} \cap M}\right)}$ is nondegenerate. Thus $\bar{q}_{\mid \overline{\left(U^{\perp}\right)}}$ is also nondegenerate.

1.4. A Coxeter arrangement over $\mathbf{F}$ is a triple $A=(V, G, q)$, where $V$ is a finite dimensional F-vector space, $G$ a finite subgroup of GL $(V)$ generated by reflections, and $q$ a $G$-invariant nondegenerate symmetric bilinear form on $V$. By a reflection we mean an endomorphism of $V$ of order 2 , which fixes pointwise some hyperplane. 
The finite set of reflection hyperplanes defines a central hyperplane arrangement which we denote by $\mathcal{A}_{G}$. A Coxeter arrangement $A=(V, G, q)$ over $\mathbf{F}$ will be called liftable if there exists an embedding $T \hookrightarrow \mathbf{C}$ of a discrete valuation ring $T$ with residue field $\mathbf{F}$ and fraction field of finite degree over $\mathbf{Q}$, and a Coxeter arrangement $A_{T}=\left(M_{T}, G_{T}, q_{T}\right)$ over $T$ such that $V$ is isomorphic to $M_{T} \otimes_{T} \mathbf{F}$ and, after identification of $M_{T} \otimes_{T} \mathbf{F}$ with $V, G=\bar{G}_{T}$ and $q=\bar{q}_{T}$. Here we denote the symmetric bilinear form induced by $q_{T}$ on $V$ by $\bar{q}_{T}$. Such a Coxeter arrangement $A_{T}$ will be called a lifting of $A$ over $T$.

The following statement is a direct consequence of Proposition 1.3.1.

Corollary 1.4. Let $T$ be a discrete valuation ring with residue field $\mathbf{F}$ and fraction field of finite degree over $\mathbf{Q}$. Fix an embedding $T \hookrightarrow$ C. Let $A=(M, G, q)$ be a Coxeter arrangement over $T$. Suppose that $p$ does not divide $|G|$ and that $\bar{q}$, the symmetric bilinear form induced by $q$ on $\bar{V}$, is nondegenerate. Then $\bar{A}:=(\bar{V}, \bar{G}, \bar{q})$ is a liftable Coxeter arrangement over $\mathbf{F}$. Furthermore the arrangement $\mathcal{A}_{G}$ has good reduction and we have $\overline{\mathcal{A}_{G}}=\mathcal{A}_{\bar{G}}$.

1.5. Let $A=(V, G, q)$ be a Coxeter arrangement over $\mathbf{F}$. Let us denote by $S(V)$ the symmetric algebra of $V$ and by $R(V)$ the subalgebra of $G$-invariants. If $p$ does not divide $|G|$, by Chevalley's Theorem ([4] Ch.V, $\S 5$ Théorème 4$), R(V)$ is generated as an $\mathbf{F}$-algebra by $n:=\operatorname{dim} V$ algebraically independent homogeneous polynomials (together with 1 ). The degrees of these polynomials are called the degrees $d_{1}, \ldots, d_{n}$ of $A$ (or the degrees of $G$ ). Their product being equal to $|G|$, they are also prime to $p$ (loc. cit. p.115).

Proposition 1.5. Let $A=(V, G, q)$ be a liftable Coxeter arrangement over $\mathbf{F}$. Assume that $p$ does not divide $|G|$. The degrees of $A$ coincide with those of any lifting of $A$ viewed as a complex Coxeter arrangement.

Proof. Let $T \subset \mathbf{C}$ be a discrete valuation ring having $\mathbf{F}$ as residue field and let $A_{T}$ be a lifting of $A$ over $T$. We have to prove that the degrees of $A$ and $A_{T}$ (viewed as a complex Coxeter arrangement) are the same. For that we may clearly suppose that $T$ is complete. Let $p_{1}, \ldots, p_{n}$ be homogeneous generators of $R(V)$. As $p$ does not divide $|G|, p_{1}, \ldots, p_{n}$ can clearly be lifted, by averaging, to elements $q_{1}, \ldots, q_{n}$ of $R\left(M_{T}\right)$, the subalgebra of $G$-invariants of the symmetric algebra of $M_{T}$. Let $\pi$ be a uniformizing parameter of $T$. The polynomials $q_{1}, \ldots, q_{n}$ generate the algebra $R\left(M_{T}\right)$ modulo $\pi$, hence, by induction, modulo $\pi^{i}$ for any $i$. Passing to the limit, we see that $q_{1}, \ldots, q_{n}$ generate the algebra $R\left(M_{T}\right)$, which implies the result.

1.6. By the following proposition, we have liftability of Coxeter arrangements over finite fields, as soon as the characteristic does not divide the order of the Coxeter group.

Proposition 1.6. Let $A=(V, G, q)$ be a Coxeter arrangement over $\mathbf{F}$. If $p$ does not divide $|G|$, then $A$ is liftable.

Proof. Since the group ring $\mathbf{F}[G]$ is semisimple, each simple component of the $\mathbf{F}[G]$ module $V$ is a direct summand of $\mathbf{F}[G]$. Thus $V$ is a projective $\mathbf{F}[G]$-module, and can hence be lifted to an $T[G]$-module $M$, of finite rank over $T$, for some discrete valuation ring $T \subset \mathbf{C}$, with residue field $\mathbf{F}$ and fraction field $K$ of finite degree over Q. In fact, by [25] $\S 14.4$ Proposition 42 , we can lift $V$ to an $R[G]$-module $M$, of finite rank over $R$, for some complete discrete valuation $\operatorname{ring} R \subset \mathbf{C}$, with residue 
field $\mathbf{F}$, and it follows from a result of M. Greenberg [14] that we can work with a henselian discrete valuation ring instead of a complete one as in loc. cit. [As was suggested by the referee one could also observe that the group algebra $\mathbf{Z}\left[\frac{1}{|G|}\right][G]$ is an Azumaya algebra over its center which is unramified over $\mathbf{Z}\left[\frac{1}{|G|}\right]$, and take $T$ to split them (cf. [20] Ch.IV §1).] Since the reduction modulo $p$ is injective on the roots of unity of order dividing $|G|$, we see that the reflections in $G \subset \operatorname{GL}(V)$ can be lifted to reflections in $\operatorname{GL}(M)$. By averaging, the form $q$ can be lifted to a $G$-invariant nondegenerate symmetric bilinear form $q_{T}$ on $M$. Moreover, the action of $G$ on $M \otimes_{T} \mathbf{C}$ can be realized over $\mathbf{R}$ (see, e.g., [25] $§ 13.2$ Théorème 31) in such a way that $q_{T}$ corresponds to a positive definite symmetric bilinear form over $\mathbf{R}$ (indeed, this follows from the proof in loc. cit.). Thus $\left(M, G, q_{T}\right)$ is a Coxeter arrangement over $T$.

\section{Character sums associated to invariant Central arrangements}

2.0. The data. Let $p$ be a prime number different from 2 and let $\mathbf{F}$ be a finite field of characteristic $p$. We denote by $\overline{\mathbf{F}}$ a fixed algebraic closure of $\mathbf{F}$. Let $f=\prod_{i \in I} \ell_{i}$ be a product of (not necessarly distinct) linear forms $\ell_{i}$ on $\mathbf{A}_{\mathbf{F}}^{n}$. The locus of $f=0$ is an arrangement $\mathcal{A}$. We denote by $U$ its complement in $\mathbf{A}_{\mathbf{F}}^{n}$. Let $q$ be a quadratic form on $\mathbf{A}_{\mathrm{F}}^{n}$ whose restriction to any stratum of $\mathcal{A}$ is nondegenerate. Choose a prime number $\ell \neq p$. Let $\chi$ be a multiplicative character $\mathbf{F}^{\times} \rightarrow \overline{\mathbf{Q}}_{\ell}^{\times}$and let $\psi$ be a nontrivial additive character $\mathbf{F} \rightarrow \overline{\mathbf{Q}}_{\ell}^{\times}$. (Here $\overline{\mathbf{Q}}_{\ell}$ denotes an algebraic closure of the field of $\ell$-adic numbers.) We denote by $\mathcal{L}_{\chi}$ and $\mathcal{L}_{\psi}$ the corresponding Kummer and Artin-Schreier sheaves (see [26] Sommes trig.). Let $G$ be a finite group, leaving $f$ and $q$ invariant, and acting freely on $U$. We consider the exponential sum

$$
\begin{aligned}
S_{G}(\chi) & =\sum_{x \in(U / G)(\mathbf{F})} \chi(f(x)) \psi(q(x)) \\
& =\operatorname{Tr}\left(F,\left(H_{c}^{\cdot}\left(U_{\overline{\mathbf{F}}}, f^{*} \mathcal{L}_{\chi} \otimes q^{*} \mathcal{L}_{\psi}\right)\right)^{G}\right) .
\end{aligned}
$$

Here $F$ denotes the geometric Frobenius automorphism and the second equality follows from Grothendieck's trace formula, see loc. cit.

At this point we should perhaps recall how the action of $G$ on the cohomology groups $H_{c}\left(U_{\overline{\mathbf{F}}}, f^{*} \mathcal{L}_{\chi} \otimes q^{*} \mathcal{L}_{\psi}\right)$ is defined. It is induced by the canonical isomorphism

$$
H_{c}^{\cdot}\left(U_{\overline{\mathbf{F}}}, f^{*} \mathcal{L}_{\chi} \otimes q^{*} \mathcal{L}_{\psi}\right) \simeq H_{c}^{\cdot}\left((U / G)_{\overline{\mathbf{F}}}, \pi_{*} \overline{\mathbf{Q}}_{\ell} \otimes f_{G}^{*} \mathcal{L}_{\chi} \otimes q_{G}^{*} \mathcal{L}_{\psi}\right)
$$

where $\pi: U \rightarrow U / G$ is the natural map, $f_{G}$ and $q_{G}: U / G \rightarrow \mathbf{A}^{1}$ are induced by $f$ and $q$, with the natural action of $G$ on $\pi_{*} \overline{\mathbf{Q}}_{\ell}$ and the trivial action on $f_{G}^{*} \mathcal{L}_{\chi}$ and $q_{G}^{*} \mathcal{L}_{\psi}$.

2.1. Concentration of the cohomology in the middle dimension. We construct the following compactification $\tilde{q}$ of $q$. Let $\Gamma$ be the closure in $\mathbf{P}^{n} \times \mathbf{A}^{1}$ of the graph of $q$ in $\mathbf{A}^{n} \times \mathbf{A}^{1}$ and let $\tilde{q}$ be the projection $\Gamma \subset \mathbf{P}^{n} \times \mathbf{A}^{1} \rightarrow \mathbf{A}^{1}$. Clearly $\tilde{q}$ is proper. We denote by $j: U \rightarrow \Gamma$ the canonical open immersion. Let $\mathcal{F}=j ! f^{*} \mathcal{L}_{\chi}$.

Lemma 2.1.1. Outside $0 \in \mathbf{A}^{n} \subset \Gamma$, the morphism $\tilde{q}$ is locally acyclic ${ }^{1}$ with respect to the sheaf $\mathcal{F}$ and $\Gamma$ is smooth over $\mathbf{F}$.

\footnotetext{
${ }^{1}$ For the notion of "locally acyclic", see [26] Th. finitude 2.12 .
} 
Proof. We look locally at $a \in \Gamma \backslash\{0\}$.

Case (i): $a \in \mathbf{A}^{n} \subset \Gamma$. We may assume that the linear forms $\ell_{i}$ which vanish at $a$ are $x_{n}, x_{n-1}, \ldots, x_{r}$ and linear combinations of these, with $r>1$. The restriction of $q$ to $x_{n}=x_{n-1}=\cdots=x_{r}=0$ is nondegenerate and $a \neq 0$, thus the restriction of $q$ is nonsingular at $a$. Hence $x_{n}, x_{n-1}, \ldots, x_{r}, q-q(a)$ are part of a system of local parameters for $\Gamma$ at $a$. In the diagram

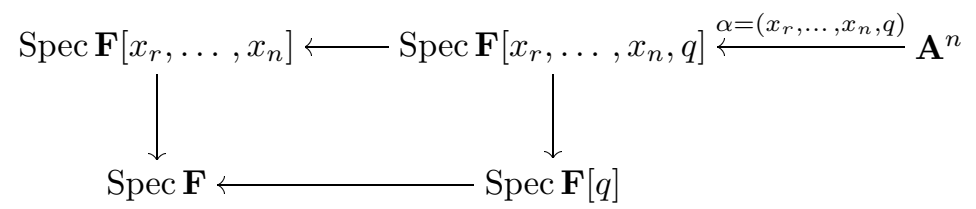

the square is cartesian, the map $\alpha$ is smooth at $a$ and, locally at $a$ for the etale topology, the sheaf $\mathcal{F}$ is the pull back of a sheaf on $\operatorname{Spec} \mathbf{F}\left[x_{r}, \ldots, x_{n}\right]$, thus $q$ is locally acyclic for $\mathcal{F}$ at $a$.

Case (ii): $a \notin \mathbf{A}^{n} \subset \Gamma$. The equation for $\Gamma$ in $\mathbf{P}^{n} \times \mathbf{A}^{1}$ is $y x_{0}^{2}=q\left(x_{1}, \ldots, x_{n}\right)$, where $x_{0}, x_{1}, \ldots, x_{n}$ are projective coordinates on $\mathbf{P}^{n}$ and $y$ is the coordinate on $\mathbf{A}^{1}$. The map $\tilde{q}$ is given by $(x, y) \mapsto y$. Let the coordinates of $a$ be $x_{i}=\alpha_{i}$ and $y=b$. Since $a \notin \mathbf{A}^{n}$, we have $\alpha_{0}=0$. Hence $q\left(\alpha_{1}, \ldots, \alpha_{n}\right)=0$. We may assume that, e.g., $\alpha_{1} \neq 0$. We may assume also that the linear forms $\ell_{i}$ which vanish at $\left(\alpha_{1}, \ldots, \alpha_{n}\right)$ are $x_{n}, x_{n-1}, \ldots, x_{r}$ and linear combinations of these, with $r>1$. Since the restriction of $q$ to $x_{n}=x_{n-1}=\cdots=x_{r}=0$ is nondegenerate, $\left(\alpha_{1}, \ldots, \alpha_{n}\right)$ is a nonsingular projective point of the projective quadric $q\left(x_{1}, \ldots, x_{r-1}, 0, \ldots, 0\right)=0$ and $r>2$. Hence $\left(1, \frac{\alpha_{2}}{\alpha_{1}}, \frac{\alpha_{3}}{\alpha_{1}}, \ldots\right)$ is a nonsingular point of the affine quadric $q\left(1, t_{2}, \ldots, t_{r-1}, 0, \ldots, 0\right)=0$. Moreover, $\Gamma$ is defined in a neighbourhood of $a$ by the affine equation $y t_{0}^{2}=q\left(1, t_{2}, \ldots, t_{n}\right)$. Here $t_{0}, t_{2}$, $\ldots, t_{n}$ are coordinates on $\mathbf{A}^{n}$ and $y$ is the coordinate on $\mathbf{A}^{1}$. We see that $t_{0}, y-b$, $t_{r}, \ldots, t_{n}$ are part of a system of local parameters for $\Gamma$ at $a$. Since $\Gamma \backslash U$ is a hypersurface of $\Gamma$ given locally at $a$ by an equation only involving $t_{0}, t_{r}, \ldots, t_{n}$, we may conclude as in case (i) that $\tilde{q}$ is locally acyclic for $\mathcal{F}$ at $a$.

Proposition 2.1.2. Assume that the sheaves $R^{i} q_{\mid U !} f^{*} \mathcal{L}_{\chi}$ have tame ramification at $\infty$ for all integers $i$. Then

$$
H_{c}^{i}\left(U_{\overline{\mathbf{F}}}, f^{*} \mathcal{L}_{\chi} \otimes q^{*} \mathcal{L}_{\psi}\right)=0 \quad \text { if } \quad i \neq n .
$$

Proof. It is a direct consequence of Lemma 2.1.1 and of a straightforward adaptation of [8] Proposition 3.1. Indeed part 3.1.1 of that proposition remains valid when we

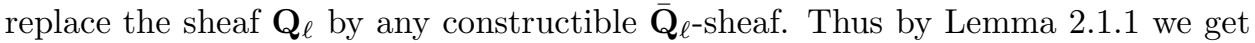
$H_{c}^{i}\left(U_{\overline{\mathbf{F}}}, f^{*} \mathcal{L}_{\chi} \otimes q^{*} \mathcal{L}_{\psi}\right)=H_{c}^{i}\left(\Gamma_{\overline{\mathbf{F}}}, \mathcal{F} \otimes \tilde{q}^{*} \mathcal{L}_{\psi}\right)=0$ when $i>n$ provided that the sheaves $R^{i} q_{\mid U !} f^{*} \mathcal{L}_{\chi}$ have tame ramification at $\infty$ for all $i$. The case $i<n$ follows by Poincaré duality and the affineness of $U$.

2.2. The case when $\chi$ is trivial. We assume throughout this paragraph that $\chi$ is trivial, thus

$$
S_{G}=\sum_{x \in(U / G)(\mathbf{F})} \psi(q(x))
$$

We also consider

$$
S=\sum_{x \in U(\mathbf{F})} \psi(q(x))=\operatorname{Tr}\left(F, H_{c}^{\cdot}\left(U_{\overline{\mathbf{F}}}, q^{*} \mathcal{L}_{\psi}\right)\right) .
$$


We may assume that the $\ell_{i}$ 's define different hyperplanes.

Lemma 2.2.1. If $\mathcal{A}=\emptyset$ and $U=\mathbf{A}^{n}$, then

$$
S=\phi(\operatorname{discr} q)(-g(\phi))^{n}
$$

where $\phi$ denotes the unique multiplicative character of $\mathbf{F}$ of order 2.

Proof. Reduce $q$ to diagonal form and use $\sum_{x \in \mathbf{A}^{1}(\mathbf{F})} \psi\left(x^{2}\right)=-g(\phi)$.

Lemma 2.2.2. The following formula holds,

$$
H_{c}^{i}\left(U_{\overline{\mathbf{F}}}, q^{*} \mathcal{L}_{\psi}\right)=0 \quad \text { if } \quad i \neq n .
$$

Proof. For $U=\mathbf{A}^{n}$, this is well known (reduce $q$ to diagonal form). The general case is a direct consequence of Lemma 3.8.1 of [8], and of the fact that $U$ is affine.

Lemma 2.2.3. The vector space $H_{c}^{n}\left(\mathbf{A}_{\overline{\mathbf{F}}}^{n}, q^{*} \mathcal{L}_{\psi}\right)$ is one dimensional and the action of an element $\sigma$ of $G$ on this space is given by multiplication by its determinant (as an operator on $\mathbf{A}^{n}$ ), $\operatorname{det} \sigma= \pm 1$.

Proof. The first statement is well known. For the second statement, we may assume $\sigma$ is a reflection since the orthogonal group is generated by reflections. By Künneth, the situation is reduced to the case where $n=1$ and then it is easy.

Notation. We set

$$
\operatorname{dim}(U, G, q):=\operatorname{dim}\left(F, H_{c}^{*}\left(U_{\overline{\mathbf{F}}}, q^{*} \mathcal{L}_{\psi}\right)^{G}\right)=\operatorname{dim}\left(F, H_{c}^{n}\left(U_{\overline{\mathbf{F}}}, q^{*} \mathcal{L}_{\psi}\right)^{G}\right)^{(-1)^{n}},
$$

with $n=\operatorname{dim} U$, and we set $\nu_{U, G}=0$ if there is a $\sigma$ in $G$ with $\operatorname{dim} \sigma \neq 1$, and $\nu_{U, G}=1$ otherwise, with $\operatorname{dim} \sigma$ denoting the determinant of the action of $\sigma$ on the affine space of $U$. Let $L(\mathcal{A})$, also denoted by $L(U)$, be the lattice of the arrangement $\mathcal{A}$. For $X \in L(\mathcal{A})$ we set $X^{\circ}=X \backslash \bigcup_{\substack{Y \in L(\mathcal{A}) \\ X \notin Y}} Y$ and $\operatorname{St}_{G}(X)=\{\sigma \in G \mid \sigma(X) \subset X\}$. We will use the above notation also when $G$ does not act freely on $U$, for example when $U$ is replaced by $X^{\circ}$ and $G$ by $\operatorname{St}_{G}(X)$. From now on till the end of subsection (2.2), we do not assume that $G$ acts freely on $U$.

Theorem 2.2.4. The following formula holds,

$$
\operatorname{dim}(U, G, q)=\left(\phi(\operatorname{discr} q) g(\phi)^{n}\right)^{(-1)^{n} \nu_{U, G}} \prod_{X \in L(U) / G} \operatorname{dim}\left(X^{\circ}, \operatorname{St}_{G}(X), q_{\mid X}\right)^{-1} .
$$

Proof. The theorem follows directly from Lemmas 2.2.1, 2.2.2 and 2.2.3 and the decomposition $U=\mathbf{A}^{n} \backslash \bigsqcup_{\bar{X} \in L(U) / G} \bigsqcup_{X \in \bar{X}} X^{\circ}$.

Notation. We denote by $\operatorname{ch}\left[\mathbf{A}^{n}, X\right]$, for $X \in L(\mathcal{A})$, the set of all chains in the lattice $L(\mathcal{A})$ which connect $\mathbf{A}^{n}$ to $X$. By definition, such a chain is a sequence $X \subset X_{1} \subset X_{2} \subset \cdots \subset X_{m} \subset \mathbf{A}^{n}$, with $X_{i}$ in $L(\mathcal{A})$, the inclusions being strict. Note that $\operatorname{St}_{G}(X)$ acts on $\operatorname{ch}\left[\mathbf{A}^{n}, X\right]$, so that we can consider the quotient $\operatorname{chcl}[X]:=$ $\operatorname{ch}\left[\mathbf{A}^{n}, X\right] / \operatorname{St}_{G}(X)$. For $c$ in $\operatorname{chcl}[X]$ we denote by $|c|$ the length of a chain representing $c$. For $X$ in $L(\mathcal{A})$, we let $\operatorname{Repr}_{X}$ be the following virtual representation of $\operatorname{St}_{G}(X)$

$$
\operatorname{Repr}_{X}:=(-1)^{n-\operatorname{dim} X} \sum_{c \in \operatorname{chcl}[X]}(-1)^{|c|-1} \operatorname{Ind}_{\mathrm{St}_{G}(c)}^{\mathrm{St}_{G}(X)}\left(\operatorname{det}_{\mid X}\right),
$$

where $\operatorname{Ind}_{\mathrm{St}_{G}(c)}^{\mathrm{St}_{G}(X)}\left(\operatorname{det}_{\mid X}\right)$ is the induced representation of the representation which to an element in $\operatorname{St}_{G}(c)$ assigns its determinant as an operator on $X$, and $\operatorname{St}_{G}(c)$ is the stabilizer of a chosen representative of $c$ in $\operatorname{ch}\left[\mathbf{A}^{n}, X\right]$. 
Theorem 2.2.5. There is an isomorphism of virtual representations of $G$,

$$
H_{c}^{n}\left(U_{\overline{\mathbf{F}}}, q^{*} \mathcal{L}_{\psi}\right) \simeq \sum_{X \in L(\mathcal{A}) / G} \operatorname{Ind}_{\mathrm{St}_{G}(X)}^{G}\left(\operatorname{Repr}_{X}\right) .
$$

Moreover this isomorphism preserves the Frobenius action if we let it act by multiplication by $\left(\phi\left(\operatorname{discr} q_{\mid X}\right)\right) g(\phi)^{\operatorname{dim} X}$ on $\operatorname{Repr}_{X}$.

Proof. By using the same decomposition of $U$ as in the proof of Theorem 2.2.4, we deduce from Lemma 2.2.2 the following isomorphism of virtual representations of $G$,

$$
H_{c}^{n}\left(U_{\overline{\mathbf{F}}}, q^{*} \mathcal{L}_{\psi}\right) \simeq \operatorname{Repr}_{\mathbf{A}^{n}}-(-1)^{n} \sum_{\substack{X \in L(\mathcal{A}) / G \\ X \neq \mathbf{A}^{n}}} \operatorname{Ind}_{\mathrm{St}_{G}(X)}^{G}(-1)^{\operatorname{dim} X} H_{c}^{\operatorname{dim} X}\left(X_{\overline{\mathbf{F}}}^{\circ}, q^{*} \mathcal{L}_{\psi}\right),
$$

which is compatible with the Frobenius action. The theorem follows now from Lemmas 2.2.1 and 2.2.3, by recursion and transitivity of induction.

The remaining results in this subsection (2.2) will not be used in the rest of the paper.

Notation. For $X \in L(\mathcal{A})$, we set $d(X):=\operatorname{dim}\left(\operatorname{Repr}_{X}\right)^{\operatorname{St}_{G}(X)}$, thus

$$
d(X)=(-1)^{n-\operatorname{dim} X} \sum_{\substack{\left.c \in \operatorname{chcl}[X] \\ \operatorname{dim}_{(\operatorname{St}}(c) \mid X\right) \subset\{1\}}}(-1)^{|c|-1},
$$

where by $\operatorname{dim}\left(\operatorname{St}_{G}(c)_{\mid X}\right) \subset\{1\}$ we mean that $\operatorname{det}\left(\sigma_{\mid X}\right)=1$ for all $\sigma$ in $\operatorname{St}_{G}(c)$.

Corollary 2.2.6. The following equalities hold

$$
\begin{gathered}
\operatorname{det}\left(F, H_{c}^{n}\left(U_{\overline{\mathbf{F}}}, q^{*} \mathcal{L}_{\psi}\right)^{G}\right)=\prod_{X \in L(\mathcal{A}) / G}\left(\phi\left(\operatorname{discr}\left(q_{\mid X}\right)\right) g(\phi)^{\operatorname{dim} X}\right)^{d(X)} \\
\operatorname{dim}\left(H_{c}^{n}\left(U_{\overline{\mathbf{F}}}, q^{*} \mathcal{L}_{\psi}\right)^{G}\right)_{\text {weight } k}=\sum_{\substack{X \in L(\mathcal{A}) / G \\
\operatorname{dim} X=k}} d(X) . \square
\end{gathered}
$$

Proposition 2.2.7. The virtual representation $\operatorname{Repr}_{X}$ is in fact a representation of $\operatorname{St}_{G}(X)$, and thus, in particular, $d(X) \geq 0$, for any $X$ in $L(\mathcal{A})$.

Proof. Let $\mathcal{A}_{X}$ be the arrangement in $\mathbf{A}^{n}$ consisting of all hyperplanes of $\mathcal{A}$ which contain $X$. Clearly $\operatorname{St}_{G}(X)$ acts on $\mathcal{A}_{X}$. Let $U_{X}$ be the complement of $\mathcal{A}_{X}$ in $\mathbf{A}^{n}$. From Theorem 2.2.5 applied to $\mathcal{A}_{X}$ we deduce that $\operatorname{Repr}_{X}$ is isomorphic, as a virtual representation of $\operatorname{St}_{G}(X)$, to $H_{c}^{n}\left(\left(U_{X}\right)_{\overline{\mathbf{F}}}, q^{*} \mathcal{L}_{\psi}\right)_{\text {weight } \operatorname{dim} X}$ which is an honest representation.

Corollary 2.2.8. Suppose $\operatorname{dim} H_{c}^{n}\left(U_{\overline{\mathbf{F}}}, q^{*} \mathcal{L}_{\psi}\right)^{G}=1$. Then there is exactly one $X_{0}$ in $L(\mathcal{A}) / G$ such that $d\left(X_{0}\right) \neq 0$. Moreover, $d\left(X_{0}\right)=1$ and

$$
\operatorname{det}\left(F, H_{c}^{n}\left(U_{\overline{\mathbf{F}}}, q^{*} \mathcal{L}_{\psi}\right)^{G}\right)=\phi\left(\operatorname{discr}\left(q_{\mid X_{0}}\right)\right) g(\phi)^{\operatorname{dim} X_{0}} .
$$

Proof. The corollary is a direct consequence of Corollary 2.2.6 (2) and Proposition 2.2.7.

We will see in (4.4) that the hypothesis of Corollary 2.2.8 is satisfied for a Coxeter arrangement over $\mathbf{F}$ when $p$ does not divide $|G|$. 
2.3. The case when $\chi=\phi$. We keep the same notations as in (2.1) and (2.2).

Lemma 2.3.1. Suppose that $f$ is the square of a polynomial over $\mathbf{F}$ on which $G$ acts by multiplication by the determinant.

(1) There is a canonical isomorphism

$$
H_{c}^{\cdot}\left((U / G)_{\overline{\mathbf{F}}}, f_{G}^{*} \mathcal{L}_{\phi} \otimes q_{G}^{*} \mathcal{L}_{\psi}\right) \simeq H_{c}^{\cdot}\left(U_{\overline{\mathbf{F}}}, q^{*} \mathcal{L}_{\psi}\right)^{\operatorname{dim}}
$$

where the superscript det denotes the part on which $G$ acts by multiplication by the determinant.

(2) For $i \neq n, H_{c}^{i}\left((U / G)_{\overline{\mathbf{F}}}, f_{G}^{*} \mathcal{L}_{\phi} \otimes q_{G}^{*} \mathcal{L}_{\psi}\right)=0$.

(3) The vector space $H_{c}^{n}\left((U / G)_{\overline{\mathbf{F}}}, f_{G}^{*} \mathcal{L}_{\phi} \otimes q_{G}^{*} \mathcal{L}_{\psi}\right)_{\text {weight } n}$ is one dimensional, and the Frobenius acts on it by multiplication by $\phi(\operatorname{discr} q) g(\phi)^{n}$.

Proof. Let us prove (1). Let $G^{+}=\{\sigma \in G \mid \operatorname{dim} \sigma=1\}$. We may suppose $G^{+} \neq G$, otherwise the assertion is trivial. We factor $\pi: U \rightarrow U / G$ into $\pi_{1}: U \rightarrow U / G^{+}$ and $\pi_{2}: U / G^{+} \rightarrow U / G$. Note that $\pi_{2}$ is the Kummer cover obtained by taking the square root of $f$, so we have canonical isomorphisms $f_{G}^{*} \mathcal{L}_{\phi} \simeq\left(\pi_{2 *} \overline{\mathbf{Q}}_{\ell}\right)^{-} \simeq$ $\left(\pi_{2 *} \overline{\mathbf{Q}}_{\ell}\right)^{\text {det }}$. From the canonical isomorphisms $\left(\pi_{2 *} \overline{\mathbf{Q}}_{\ell}\right)^{\text {det }} \simeq\left(\pi_{2 *}\left(\pi_{1 *} \overline{\mathbf{Q}}_{\ell}\right)^{G^{+}}\right)^{\text {det }} \simeq$ $\left(\left(\pi_{2 *} \pi_{1 *} \overline{\mathbf{Q}}_{\ell}\right)^{G^{+}}\right)^{\text {det }} \simeq\left(\pi_{*} \overline{\mathbf{Q}}_{\ell}\right)^{\text {det }}$, we obtain canonical isomorphisms

$$
\begin{aligned}
H_{c}^{\cdot}\left((U / G)_{\overline{\mathbf{F}}}, f_{G}^{*} \mathcal{L}_{\phi} \otimes q_{G}^{*} \mathcal{L}_{\psi}\right) & \simeq H_{c}^{\cdot}\left((U / G)_{\overline{\mathbf{F}}},\left(\pi_{*} \overline{\mathbf{Q}}_{\ell}\right)^{\operatorname{det}} \otimes q_{G}^{*} \mathcal{L}_{\psi}\right) \\
& \simeq H_{c}^{\cdot}\left((U / G)_{\overline{\mathbf{F}}},\left(\pi_{*} \overline{\mathbf{Q}}_{\ell}\right) \otimes q_{G}^{*} \mathcal{L}_{\psi}\right)^{\operatorname{det}} \\
& \simeq H_{c}^{\cdot}\left(U_{\overline{\mathbf{F}}}, q_{G}^{*} \mathcal{L}_{\psi}\right)^{\operatorname{det}}
\end{aligned}
$$

The assertion (2) is a direct consequence of (1) and Lemma 2.2.2, while (3) follows directly from (1) and Theorem 2.2.5.

Proposition 2.3.2. Suppose that the assumptions of Lemma 2.3.1 are valid and that $H_{c}^{n}\left(U_{\overline{\mathbf{F}}}, f^{*} \mathcal{L}_{\phi} \otimes q^{*} \mathcal{L}_{\psi}\right)^{G}$ is of dimension 1. Then

$$
S_{G}(\phi)=(-1)^{n} \phi(\operatorname{discr} q) g(\phi)^{n} .
$$

Proof. It is a direct consequence of the preceding lemma.

\section{Monodromy And CRitical VAlues for Coxeter ARrangements}

3.1. Let $(V, G, q)$ be a Coxeter arrangement over $\mathbf{C}$. For each reflection hyperplane $H$, we choose a linear form $\ell_{H}: V \rightarrow \mathbf{C}$ defining $H$ in $V$ and we set $\delta=\left(\prod_{H} \ell_{H}\right)^{2}$. We denote by $N$ the number of reflection hyperplanes and by $\Delta: V / G \rightarrow \mathbf{C}$ the map induced by $\delta$. Thus $\Delta$ is the discriminant of $G$. We denote by $F_{0}$ the Milnor fiber of $\Delta$ at 0 and by $Z(T, G)$ the zeta function of local monodromy of $\Delta$ at 0 , i.e.,

$$
Z(T, G)=\prod_{i} \operatorname{det}\left(1-T M, H^{i}\left(F_{0}, \mathbf{C}\right)\right)^{(-1)^{i+1}},
$$

where $M$ denotes the monodromy automorphism (see, e.g., [3]).

Let $d_{1}, \ldots, d_{n}$ be the degrees of $G$. In [10] we proved the following result.

Theorem 3.1. For $G$ a finite Coxeter group we have

$$
\prod_{\mathcal{E} \text { connected subgraph }} Z(-T, G(\mathcal{E}))^{(-1)^{|\mathcal{E}|}}=\prod_{i=1}^{n} \frac{1-T^{d_{i}}}{1-T},
$$

where the product on the left-hand side runs over all connected subgraphs $\mathcal{E}$ of the Coxeter diagram of $G, G(\mathcal{E})$ denotes the Coxeter group with diagram $\mathcal{E}$, and $|\mathcal{E}|$ the number of vertices of $\mathcal{E}$. 
The proof of Theorem 3.1 given in [10] depends on a case by case analysis. A more conceptual proof, but depending on Macdonald's formula (0.1), is given in [7].

We use now terminology from [27] II Exp. XIII - XIV. Denote by $\bar{\eta}_{0}$ the generic geometric point of the henselization of $\mathbf{A}_{\mathbf{C}}^{1}$ at 0 and by $I_{0, \mathbf{C}}$ its inertia group (i.e., the fundamental group of the complement of 0 in a small disk around 0 ). We denote by $K_{I_{0}, \mathbf{C}}$ the Grothendieck group of finite dimensional vector spaces with $I_{0, \mathbf{C}}$-action. If $\mathcal{L}$ is an object in $D_{c}^{b}\left(\mathbf{G}_{m, \mathbf{C}}, \mathbf{C}\right)$, we denote by $\left[\mathcal{L}_{\bar{\eta}_{0}}\right]$ the class of $\sum(-1)^{i}\left[\mathcal{H}^{i}(\mathcal{L})_{\bar{\eta}_{0}}\right]$ in $K_{I_{0, \mathrm{C}}}$ and we set $\left[\mathcal{L}_{\bar{\eta}_{\infty}}\right]=\left[\operatorname{inv}^{*}(\mathcal{L})_{\bar{\eta}_{0}}\right]$, where inv is the morphism $x \mapsto x^{-1}$. If a finite group $G$ acts on $\mathcal{L}$, we denote by $\left[\mathcal{L}_{\bar{\eta}_{0}}^{G}\right]$ the class of $\sum(-1)^{i}\left[\mathcal{H}^{i}(\mathcal{L})_{\bar{\eta}_{0}}^{G}\right]$ and we define similarly $\left[\mathcal{L}_{\bar{\eta}_{\infty}}^{G}\right]$. For any character $\chi: I_{0, \mathbf{C}} \rightarrow \mathbf{C}^{\times}$we denote by $V_{\chi}$ the class in $K_{I_{0, \mathbf{C}}}$ of the rank one object with action given by $\chi$ and for any natural number $m \geq 1$, we set $V_{m}=\left[\left(\pi_{m *} \mathbf{C}\right)_{\bar{\eta}_{0}}\right]$, where $\pi_{m}: \mathbf{G}_{m} \rightarrow \mathbf{G}_{m}$ is given by $x \mapsto x^{m}$. Of course, we have $V_{m}=\sum_{\chi^{m}=1} V_{\chi}$. We set $\bar{M}_{G}=(-1)^{n-1}\left[\left(R \psi_{\Delta}(\mathbf{C})\right)_{0}\right]$. Here $\left(R \psi_{\Delta}(\mathbf{C})\right)_{0}$ is the stalk at zero of the complex of nearby cycles with respect to $\Delta$.

One can rephrase Theorem 3.1 as follows.

Theorem 3.1'. For $G$ a finite Coxeter group, the following equality in $K_{I_{0, \mathbf{C}}}$ holds,

$$
\sum_{\mathcal{E} \text { connected subgraph }} \bar{M}_{G(\mathcal{E})}=V_{\phi} \otimes\left(\sum_{i=1}^{n}\left(V_{d_{i}}-V_{1}\right)\right),
$$

where $\phi$ is the unique character of order 2.

3.2. Let $U$ be the complement in $V$ of $\delta=0$, let $B$ (resp. $B_{0}$ ) be the intersection of $U$ with the locus of $q=1$ (resp. $q=0$ ). The following proposition is proved in [7] by a detailed analysis of the geometry of a nice compactification of $U$. Here and in the following we denote by $q_{\mid U}^{N}$ the restriction of the map defined by $q$ on $U$ raised to the $N$-th power.

Proposition 3.2.1. We have the following equalities in $K_{I_{0, \mathrm{C}}}$,

(1) $\left[\left(R \delta_{\mid B_{0} !} \mathbf{C}\right)_{\bar{\eta}_{\infty}}^{G}\right]=\left[\left(R \delta_{\mid B !} \mathbf{C}\right)_{\bar{\eta}_{\infty}}^{G}\right]$.

(2) $\left[\left(R \delta_{\mid B !} \mathbf{C}\right)_{\bar{\eta}_{\infty}}^{G}\right]+\left[\left(R q_{\mid U !}^{N} \mathbf{C}\right)_{\bar{\eta}_{0}}^{G}\right]=\left[\left(R \psi_{\Delta}(\mathbf{C})\right)_{0}\right]$.

(3) $\left[\left(R \delta_{\mid B !} \mathbf{C}\right)_{\bar{\eta}_{0}}^{G}\right]=(-1)^{n} \sum \mathcal{E} \begin{gathered}\text { connected subgraph } \\ G(\mathcal{E}) \neq G\end{gathered} \bar{M}_{G(\mathcal{E})}$.

(4) There exist $\bar{a}, \bar{b}$ in $\mathbf{Z}$ with $\bar{a}+\bar{b}=(-1)^{n-1}$ such that $\left[\left(R q_{\mid U !}^{N} \mathbf{C}\right)_{\bar{\eta}_{0}}^{G}\right]=(\bar{a}-\bar{b}) V_{N}+$ $\bar{b} V_{2 N}$ and such that $\left[\left(R q_{\mid U !} \delta^{*} \mathcal{L}_{\chi}\right)_{\bar{\eta}_{0}}^{G}\right]=\bar{a} V_{\chi^{N}}+\bar{b} V_{\phi \chi^{N}}$, for any character $\chi$ of $I_{0, \mathbf{C}}$, where $\mathcal{L}_{\chi}$ denotes the Kummer sheaf associated to $\chi$.

Corollary 3.2.2. The equality

$$
\left[\left(R \delta_{\mid B !} \mathbf{C}\right)_{\bar{\eta}_{0}}^{G}\right]-\left[\left(R \delta_{\mid B !} \mathbf{C}\right)_{\bar{\eta}_{\infty}}^{G}\right]-\left[\left(R q_{\mid U !}^{N} \mathbf{C}\right)_{\bar{\eta}_{0}}^{G}\right]=(-1)^{n}\left(V_{\phi} \otimes \sum_{i=1}^{n}\left(V_{d_{i}}-V_{1}\right)\right)
$$

holds in $K_{I_{0, \mathbf{C}}}$.

Proof. The corollary follows directly from (2), (3) and Theorem 3.1'.

Actually, the values of $\bar{a}$ and $\bar{b}$ are determined by the following proposition, which is proved in [7], but not needed in the present paper.

Proposition 3.2.3. The following formula holds,

$$
\left[\left(R q_{\mid U !} \mathbf{C}\right)_{\bar{\eta}_{0}}^{G}\right]=(-1)^{n-1} V_{\phi^{n+N}}=\bar{a} V_{1}+\bar{b} V_{\phi}
$$


3.3. If $f: X \rightarrow \mathbf{G}_{m, \mathbf{C}}$ is a morphism of schemes of finite type over $\mathbf{C}$, we set $a(f)=\prod_{s \in \mathbf{G}_{m, \mathbf{C}}} s^{a_{s}\left(R f_{!} \mathbf{C}\right)}$, where $a_{s}$ is the drop of the rank of $R f_{!}(\mathbf{C})$ at $s$ (i.e., the alternating sum of the drops of the rank of its cohomology sheaves, $c f$. [16] 3.1.5.2. We denote by $\Delta_{B}$ the function $B / G \rightarrow \mathbf{G}_{m, \mathbf{C}}$ induced by $\delta$. We will use the following result which is proven in [7] as an easy consequence of Macdonald's formula (0.1), by which one calculates the critical value (0.5) of $\Delta_{B}$.

Theorem 3.3. The following formula holds,

$$
a\left(\Delta_{B}\right)=\left(\kappa \frac{\prod_{i} d_{i}^{d_{i}}}{N^{N}}\right)^{(-1)^{n}},
$$

where $\kappa$ is as in $(0.2)$.

\section{Proof of the Main Theorem}

4.1. Determinants and monodromy. Let $U$ be a scheme of finite type over the finite field $\mathbf{F}$. We assume that the characteristic $p$ of $\mathbf{F}$ is not equal to 2 and we fix a prime $\ell$ distinct from $p$. We denote by $\overline{\mathbf{F}}$ an algebraic closure of $\mathbf{F}$ and we set $U_{\overline{\mathbf{F}}}=U \otimes_{\mathbf{F}} \overline{\mathbf{F}}$. For any object $\mathcal{F}$ in $D_{c}^{b}\left(U, \overline{\mathbf{Q}}_{\ell}\right)$ (the derived category of "bounded complexes" of $\overline{\mathbf{Q}}_{\ell}$-sheaves with constructible cohomology), we denote

$$
\varepsilon_{0}(U / \mathbf{F}, \mathcal{F}):=\operatorname{det}\left(-F, H_{c}^{\cdot}\left(U_{\overline{\mathbf{F}}}, \mathcal{F}\right)\right)^{-1} .
$$

Let $f: U \rightarrow \mathbf{G}_{m, \mathbf{F}}$ be a morphism. We set $a(f)=\prod_{s \in \mathbf{G}_{m, \overline{\mathbf{F}}}} s^{a_{s}\left(R f_{!} \overline{\mathbf{Q}}_{\ell}\right)} \in \mathbf{F}$, where $a_{s}$ denotes the total drop at $s$ of a complex of sheaves (cf. [16] 3.1.5.2). We also denote by $\bar{\eta}_{0}$ the generic geometric point of the henselization of $\mathbf{A}_{\overline{\mathbf{F}}}^{1}$ at 0 and by $I_{0}\left(\right.$ or $\left.I_{0, \overline{\mathbf{F}}}\right)$ the inertia group. We denote by $K_{I_{0}}$ the Grothendieck group of finite dimensional $\overline{\mathbf{Q}}_{\ell}$-vector spaces with continuous action of $I_{0}$ defined on a finite extension of $\mathbf{Q}_{\ell}$. For $\mathcal{L}$ an object in $D_{c}^{b}\left(\mathbf{G}_{m, \mathbf{F}}, \overline{\mathbf{Q}}_{\ell}\right)$ we define as in (3.1) objects $\left[\mathcal{L}_{\bar{\eta}_{0}}\right]$ and $\left[\mathcal{L}_{\bar{\eta}_{\infty}}\right]$ in $K_{I_{0}}$. Also if a finite group $G$ acts on $\mathcal{L}$, we define similarly $\left[\mathcal{L}_{\bar{\eta}_{0}}^{G}\right]$ and $\left[\mathcal{L}_{\bar{\eta}_{\infty}}^{G}\right]$. For $\chi: I_{0} \rightarrow \overline{\mathbf{Q}}_{\ell}^{\times}$a continuous character, we denote by $V_{\chi}$ the associated object in $K_{I_{0}}$. For $N \geq 1$ an integer, we define $V_{N}$ in $K_{I_{0}}$ as in (3.1). We will denote by $\phi$ the character of order 2 of $I_{0}$. For any character $\chi: \mathbf{F}^{\times} \rightarrow \overline{\mathbf{Q}}_{\ell}^{\times}$ we denote by $\mathcal{L}_{\chi}$ the corresponding Kummer sheaf on $\mathbf{G}_{m, \mathbf{F}}$. We can also view $\chi$ as a continuous character $I_{0} \rightarrow \overline{\mathbf{Q}}_{\ell}^{\times}$which we still denote by $\chi$.

Suppose $\left[\left(R f_{!} \overline{\mathbf{Q}}_{\ell}\right)_{\bar{\eta}_{0}}\right]=\sum_{N \in \mathbf{N}^{\times}} \alpha_{N} V_{N}$ and $\left[\left(R f_{!} \overline{\mathbf{Q}}_{\ell}\right)_{\bar{\eta}_{\infty}}\right]=\sum_{N \in \mathbf{N}^{\times}} \beta_{N} V_{N}$ with $\alpha_{N}$ and $\beta_{N}$ in $\mathbf{Z}$. (Here $\mathbf{N}^{\times}$denotes $\mathbf{N} \backslash\{0\}$.) We define the rational number $b(f)$ as

$$
b(f):=\left(\prod_{N \in \mathbf{N}^{\times}} N^{N \alpha_{N}}\right)\left(\prod_{N \in \mathbf{N}^{\times}}(-N)^{-N \beta_{N}}\right) .
$$

For any character $\chi: \mathbf{F}^{\times} \rightarrow \overline{\mathbf{Q}}_{\ell}^{\times}$we set

$$
G(f, \chi)=\prod_{N \in \mathbf{N}^{\times}}\left(g\left(\chi^{N}\right)^{\alpha_{N}} g\left(\chi^{-N}\right)^{\beta_{N}}\right)
$$

and

$$
\tilde{G}(f, \chi)=\prod_{N \in \mathbf{N}^{\times}}\left(g\left(\chi^{N}\right)^{\alpha_{N}-\beta_{N}}\right)
$$

where $g(\chi)$ is the Gauss sum $g(\chi):=-\sum_{x \in \mathbf{F}^{\times}} \chi(x) \psi(x)$ for $\psi$ a fixed nontrivial additive character. 
Theorem 4.1.1. Assume that $\alpha_{N}=\beta_{N}=0$ when $p$ divides $N$ and that the sheaves $R^{i} f_{!} \overline{\mathbf{Q}}_{\ell}$ are tame at 0 and $\infty$ for every $i$. Then, for every multiplicative character $\chi$ of $\mathbf{F}^{\times}$,

$$
\begin{aligned}
\varepsilon_{0}\left(U / \mathbf{F}, f^{*} \mathcal{L}_{\chi}\right) & =\varepsilon_{0}\left(U / \mathbf{F}, \overline{\mathbf{Q}}_{\ell}\right) \chi\left(\frac{a(f)}{b(f)}\right) G(f, \chi) \\
& =\varepsilon_{0}\left(U / \mathbf{F}, \overline{\mathbf{Q}}_{\ell}\right)|\mathbf{F}|^{\sum_{\chi^{N} \neq 1} \beta_{N}} \chi\left(\frac{a(f)}{|b(f)|}\right) \tilde{G}(f, \chi) .
\end{aligned}
$$

Proof. The theorem follows directly from [9] Proposition 2.4.1, which is based on Laumon's product formula [16] 3.2.1.1, using [16] 3.1.5.4 (iv), and $g\left(\chi^{-1}\right)=$ $|\mathbf{F}| \chi(-1) g(\chi)^{-1}$ if $\chi \neq 1$. For more details, see [6] Proposition 3.2.1. A much stronger result is contained in [23].

Remark. The advantage of the second equality upon the first is that $|b(f)|$ and $\tilde{G}(f, \chi)$ only depend on $\left[\left(R f_{!} \overline{\mathbf{Q}}_{\ell}\right)_{\bar{\eta}_{0}}\right]-\left[\left(R f_{!} \overline{\mathbf{Q}}_{\ell}\right)_{\bar{\eta}_{\infty}}\right]$.

Let $\mathcal{F}$ be a constructible $\ell$-adic sheaf on a scheme $U$ (of finite type) over $\mathbf{F}$ and suppose a finite group $G$ acts on $U$ and $\mathcal{F}$. We set

$$
\varepsilon_{0}^{G}(U / \mathbf{F}, \mathcal{F}):=\operatorname{det}\left(-F, H_{c}\left(U_{\overline{\mathbf{F}}}, \mathcal{F}\right)^{G}\right)^{-1} .
$$

Notation. For $\chi$ any multiplicative character of $\mathbf{F}^{\times}$and for $F$ in $K_{I_{0, \mathbf{C}}}$ of the form $F=\sum_{N \in \mathbf{N} \times} \gamma_{N} V_{N}$, with $\gamma_{N}$ in $\mathbf{Z}$, we set

$$
\tilde{G}(F, \chi)=\prod_{N} g\left(\chi^{N}\right)^{\gamma_{N}}
$$

and

$$
b(F)=\prod_{N} N^{N \gamma_{N}} .
$$

It is assumed here that a nontrivial additive character has been fixed. Note that $\tilde{G}$ and $b$ are multiplicative in $F$.

Lemma 4.1.2. (1) If $N \in \mathbf{N}^{\times}$is even, $V_{N} \otimes V_{\phi}=V_{N}, \tilde{G}\left(V_{N} \otimes V_{\phi}, \chi\right)=\tilde{G}\left(V_{N}, \chi\right)$ and $b\left(V_{N} \otimes V_{\phi}\right)=b\left(V_{N}\right)$.

(2) If $N \in \mathbf{N}^{\times}$is odd, $V_{N} \otimes V_{\phi}=V_{2 N}-V_{N}, \tilde{G}\left(V_{N} \otimes V_{\phi}, \chi\right)=\frac{\chi\left(4^{N}\right)}{g(\phi)} \tilde{G}\left(V_{N}, \phi \chi\right)$ and $b\left(V_{N} \otimes V_{\phi}\right)=4^{N} b\left(V_{N}\right)$.

Proof. Everything is clear except perhaps the second relation in (2) which follows directly from the Hasse-Davenport formula ( $c f .[11])$ and the first one.

4.2. Fix an integer $d \geq 1$. Let $R \subset \mathbf{C}$ be a Dedekind domain containing the $d$-th roots of unity, with fraction field $K$ of finite degree over $\mathbf{Q}$. We denote by $\bar{K}=\overline{\mathbf{Q}}$ the algebraic closure of $K$ in C. Let $A=(V, G, q)$ be a Coxeter arrangement over $R$. For $\mathfrak{P}$ a maximal ideal of $R$ we denote by $\left(\bar{V}_{\mathfrak{P}}, \bar{G}_{\mathfrak{P}}, \bar{q}_{\mathfrak{P}}\right)$ the corresponding data over $k_{\mathfrak{P}}:=R / \mathfrak{P}$. When no confusion can arise, we will omit the index $\mathfrak{P}$. Choose linear forms $\ell_{H}$ over $R$ which define the hyperplanes of $\mathcal{A}_{G}$. Let $\mathfrak{P}$ be a maximal ideal of $R$ which is relatively prime to $d|G|$, such that the linear forms $\ell_{H}$ are not zero modulo $\mathfrak{P}$ and such that $\bar{q}_{\mathfrak{P}}$ is a nondegenerate bilinear form. (In particular $\mathfrak{P}$ is relatively prime to 2 and to each degree $d_{i}$ of $G$, because $|G|$ is even and equal to the product of the $d_{i}$ 's.) Thus the conclusions of Propositions 1.2.2, 1.3.1, and 1.3.3 hold. In this situation there is no ambiguity for the notations, $\delta_{\mathfrak{P}}, U_{\mathfrak{P}}, B_{\mathfrak{P}}, B_{0 \mathfrak{P}}$, etc, $c f$. the notation in 3.1 and 3.2 , which refer to objects attached to the reduction 
of $A \bmod \mathfrak{P}$, which are also the reduction $\bmod \mathfrak{P}$ of the corresponding objects over $R$. We denote by $I_{0, \bar{K}}$ the inertia group at 0 of $\mathbf{G}_{m, \bar{K}}$ and we fix a character $\chi: I_{0, \bar{K}} \rightarrow \overline{\mathbf{Q}}_{\ell}^{\times}$of order $d$. The Kummer sheaf $\mathcal{L}_{\chi}$ on $\mathbf{G}_{m, \bar{K}}$ is obtained by base change from a sheaf, which we still denote by $\mathcal{L}_{\chi}$, on $\mathbf{G}_{m, R}$. The character $\chi$ induces a character $\chi_{\mathfrak{P}}: I_{0, \bar{k}_{\mathfrak{P}}} \rightarrow \overline{\mathbf{Q}}_{\ell}^{\times}$(here $\bar{k}_{\mathfrak{P}}$ denotes an algebraic closure of $k_{\mathfrak{P}}$ ) which is associated to a character, still denoted by the same symbol, $\chi_{\mathfrak{P}}: k_{\mathfrak{P}}^{\times} \rightarrow \overline{\mathbf{Q}}_{\ell}^{\times}$. For $s$ an element of $K$ we write $\chi_{\mathfrak{P}}(s)$ for the value of $\chi_{\mathfrak{P}}$ on the residue class of $s$ if $s$ is a unit in $R_{\mathfrak{P}}$ and we set $\chi_{\mathfrak{P}}(s)=1$ otherwise.

Proposition 4.2.1. Let $\mathfrak{P}$ be a maximal ideal of $R$ satisfying the above conditions. Then $a\left(\Delta_{B}\right) / b(F)$ and $\kappa$ are units in $R_{\mathfrak{P}}$ and

$$
\begin{aligned}
\chi_{\mathfrak{P}}\left(\frac{a\left(\Delta_{B}\right)}{b(F)}\right) \tilde{G}\left(F, \chi_{\mathfrak{P}}\right) \\
\quad=C \chi_{\mathfrak{P}}(\kappa)^{(-1)^{n}} \prod_{1 \leq i \leq n}\left[\frac{g\left(\left(\phi \chi_{\mathfrak{P}}\right)^{d_{i}}\right)}{g\left(\phi \chi_{\mathfrak{P}}\right)}\right]^{(-1)^{n}} \frac{g\left(\chi_{\mathfrak{P}}^{N}\right)^{\bar{a}} g\left(\phi \chi_{\mathfrak{P}}^{N}\right)^{\bar{b}}}{g(\phi)^{\bar{b}}}
\end{aligned}
$$

for $F=\left[\left(R \delta_{\mid B !} \mathbf{C}\right)_{\bar{\eta}_{0}}^{G}\right]-\left[\left(R \delta_{\mid B !} \mathbf{C}\right)_{\bar{\eta}_{\infty}}^{G}\right]$ in $K_{I_{0, \mathbf{C}}}$ (it follows from $\S 3$ and Lemma 4.1.2 that $F$ has the required form), with $C=\left[g(\phi)^{\left(\sum_{d_{i} \text { odd }} 1\right)-n}\right]^{(-1)^{n-1}}$. Here, as usual, $\phi$ denotes the character of order 2.

Proof. It follows from Proposition 1.3.3 that $\kappa$ is a unit in $R_{\mathfrak{P}}$. By Corollary 3.2.2,

$$
F-E=(-1)^{n}\left(V_{\phi} \otimes \sum_{i=1}^{n}\left(V_{d_{i}}-V_{1}\right)\right)
$$

with $E=\left[\left(R q_{\mid U !}^{N} \mathbf{C}\right)_{\bar{\eta}_{0}}^{G}\right]$, and by 3.2.1 (4), $E=\bar{a} V_{N}+\bar{b}\left(V_{2 N}-V_{N}\right)$. So by Lemma 4.1.2, we obtain that $b(F-E)$ is a unit in $R_{\mathfrak{P}}$ and

$$
\frac{\tilde{G}\left(F-E, \chi_{\mathfrak{P}}\right)}{\chi_{\mathfrak{P}}(b(F-E))}=C \prod_{1 \leq i \leq n}\left[\frac{g\left(\left(\phi \chi_{\mathfrak{P}}\right)^{d_{i}}\right)}{g\left(\phi \chi_{\mathfrak{P}}\right)}\right]^{(-1)^{n}} \chi_{\mathfrak{P}}\left(\prod_{1 \leq i \leq n} d_{i}^{d_{i}}\right)^{(-1)^{n-1}},
$$

while $b(E) N^{(-1)^{n} N}$ is a unit in $R_{\mathfrak{P}}$ and

$$
\frac{\tilde{G}\left(E, \chi_{\mathfrak{P}}\right)}{\chi_{\mathfrak{P}}\left(b(E) N^{(-1)^{n} N}\right)}=\frac{g\left(\chi_{\mathfrak{P}}^{N}\right)^{\bar{a}} g\left(\phi \chi_{\mathfrak{P}}^{N}\right)^{\bar{b}}}{g(\phi)^{\bar{b}}}
$$

(To verify this last equation when $N$ is even, one has again to use the HasseDavenport formula as in the proof of 4.1.2.) Now the formula follows directly from the multiplicativity of $\tilde{G}$ and $b$ and from Theorem 3.3.

Proposition 4.2.2. For almost all $\mathfrak{P}$ in $\operatorname{Spec} R$ (i.e., for all but a finite number),

$$
\varepsilon_{0}^{G}\left(B_{\mathfrak{P}} / k_{\mathfrak{P}}, \delta_{\mathfrak{P}}^{*} \mathcal{L}_{\chi_{\mathfrak{P}}}\right)=\varepsilon_{0}^{G}\left(B_{\mathfrak{P}} / k_{\mathfrak{P}}, \overline{\mathbf{Q}}_{\ell}\right)\left|k_{\mathfrak{P}}\right|^{\sum_{\chi^{j} \neq 1} \beta_{j}} \chi_{\mathfrak{P}}\left(\frac{a\left(\Delta_{B}\right)}{b(F)}\right) \tilde{G}\left(F, \chi_{\mathfrak{P}}\right)
$$

and

$$
\varepsilon_{0}^{G}\left(B_{0 \mathfrak{P}} / k_{\mathfrak{P}}, \delta_{\mathfrak{P}}^{*} \mathcal{L}_{\chi_{\mathfrak{P}}}\right)=\varepsilon_{0}^{G}\left(B_{0 \mathfrak{P}} / k_{\mathfrak{P}}, \overline{\mathbf{Q}}_{\ell}\right)\left|k_{\mathfrak{P}}\right|^{\sum_{\chi^{j} \neq 1} \beta_{j}},
$$

where the $\beta_{j}$ are given by $\left[\left(R \delta_{\mid B !} \mathbf{C}\right)_{\bar{\eta}_{\infty}}^{G}\right]=\sum_{j} \beta_{j} V_{j}$. 
Proof. The first assertion is a direct consequence of Theorem 4.1.1, using standard constructibility, comparison and base change theorems. For the second assertion, note that because $\delta$ and $q$ are homogeneous the sheaves $R^{i} \delta_{\mid B_{0} !} \mathbf{C}$ are lisse on $\mathbf{G}_{m}$. Thus $\left|b\left(\Delta_{\mid B_{0} / G}\right)\right|=1$ (with $b$ defined as in 4.1$)$ and $\left[\left(R \delta_{\mid B_{0} !} \mathbf{C}\right) \bar{\eta}_{0}\right]=\left[\left(R \delta_{\mid B_{0} !} \mathbf{C}\right)_{\bar{\eta}_{\infty}}^{G}\right]$. Now the result follows similarly from Theorem 4.1.1 and Proposition 3.2.1 (1) (that $\left.\left[\left(R \delta_{\mid B_{0} !} \mathbf{C}\right)_{\bar{\eta}_{\infty}}^{G}\right]=\left[\left(R \delta_{\mid B !} \mathbf{C}\right)_{\bar{\eta}_{\infty}}^{G}\right]\right)$.

4.3. Good reduction. Let $A=(V, G, q)$ be a liftable Coxeter arrangement over F. We choose defining linear forms for each reflection hyperplane. Let $d \geq 1$ be an integer and $\chi: \mathbf{F}^{\times} \rightarrow \overline{\mathbf{Q}}_{\ell}^{\times}$a character of order $d$. There exists a discrete valuation $\operatorname{ring} T \subset \mathbf{C}$, with residue field $\mathbf{F}$ and fraction field of finite degree over $\mathbf{Q}$, containing the $d$-th roots of unity, and a lifting $A_{T}$ of $A$ over $T$. We denote by $K$ the fraction field of $T$, by $\bar{K}$ its algebraic closure in $\mathbf{C}$ and by $\mathcal{L}_{\chi}$ the Kummer sheaf on $\mathbf{G}_{m, T}$ associated to $\chi$. We assume that $p$ does not divide $|G|$ and we choose defining linear forms with coefficients in $T$ for each reflection hyperplane, which reduce to those already chosen in $\mathbf{F}$. We denote by $\delta_{T}, U_{T}, B_{T}$, etc, the data associated to $A_{T}$.

Lemma 4.3.1. If $p$ does not divide $|G|$, the specialization morphism

$$
H_{c}^{i}\left(B \otimes \overline{\mathbf{F}}, \delta^{*} \mathcal{L}_{\chi}\right) \longrightarrow H_{c}^{i}\left(B_{T} \otimes \bar{K}, \delta_{T}^{*} \mathcal{L}_{\chi}\right)
$$

is an isomorphism for all $i$.

Proof. The arrangement associated to $A_{T}$ has good reduction by Proposition 1.3.1. Thus the $T$-scheme $U_{T}$ admits a canonical smooth compactification $\bar{U}_{T}$ over $T$ such that $\bar{U}_{T} \backslash U_{T}$ has normal crossings over $T$, and such that $\bar{U}_{T}$ is equipped with a morphism $\pi: \bar{U}_{T} \rightarrow \mathbf{P}_{T}^{n}$, where $\mathbf{P}_{T}^{n}$ is the projective closure of $V_{T}$, extending the inclusion of $U_{T}$ in $V_{T}$. The compactification $\bar{U}_{T}$ and the morphism $\pi$ are obtained by blowing up successively the union of the strict transforms of the strata of dimension $i$ of the projective arrangement associated to $A_{T}$ for $i$ increasing from 0 to $n-2$ ( $c f$. $[17] \S 7$ ). We now define $\bar{B}_{T}$ to be the strict transform in $\bar{U}_{T}$ of the closure of $B_{T}$ in $\mathbf{P}_{T}^{n}$. It follows from Proposition 1.3.3 that $\bar{B}_{T}$ intersects the strata of $\bar{U}_{T} \backslash U_{T}$ transversally. Hence $\bar{B}_{T}$ is smooth and proper over $T$ and $\bar{B}_{T} \backslash B_{T}$ has normal crossings over $T$. The assertion now follows from [26] Th. finitude Appendice 1.3.3 and 2.4.

Corollary 4.3.2. Let $A=(V, G, q)$ be a liftable Coxeter arrangement over $\mathbf{F}$. If $p$ does not divide $|G|$, then

$$
\sum(-1)^{i} \operatorname{dim} H_{c}^{i}\left(B \otimes \overline{\mathbf{F}}, \delta^{*} \mathcal{L}_{\chi}\right)^{G}=(-1)^{n-1} .
$$

Proof. The isomorphism in Lemma 4.3 .1 being $G$-equivariant, we only have to prove the analogous result for Coxeter arrangements over $\mathbf{C}$. In this case

$$
\begin{aligned}
\sum(-1)^{i} \operatorname{dim} H_{c}^{i}\left(B, \delta^{*} \mathcal{L}_{\chi}\right)^{G} & =\sum(-1)^{i} \operatorname{dim} H_{c}^{i}(B, \mathbf{C})^{G} \\
& =|G|^{-1} \chi(B, \mathbf{C}) .
\end{aligned}
$$

But in [7] we proved that $\chi(B, \mathbf{C})=(-1)^{n-1}|G|$ for complex Coxeter arrangements. (The proof is by an easy induction in a more general setting, showing that $(-1)^{n-1} \chi(B, \mathbf{C})$ equals the number of Weyl chambers.)

Lemma 4.3.3. If $p$ does not divide $|G|$, the sheaves $R^{i} q_{T \mid U_{T} !} \delta_{T}^{*} \mathcal{L}_{\chi}$ are lisse on $\mathbf{G}_{m, T}$ for all $i$. 
Proof. The polynomials associated to $\delta_{T}$ and $q_{T}$ being homogeneous it is enough to prove that the specialization morphisms associated to the specialization of the generic point of $t=1$ to the special point are isomorphims, which is precisely the content of Lemma 4.3.1.

Corollary 4.3.4. Let $A=(V, G, q)$ be a liftable Coxeter arrangement over $\mathbf{F}$. If $p$ does not divide $|G|$, the sheaves $R^{i} q_{\mid U !} \delta^{*} \mathcal{L}_{\chi}$ are lisse and tame on $\mathbf{G}_{m, \mathbf{F}}$ for all $i$.

Proof. This is a direct consequence of Lemma 4.3.3, see, e.g., [8] (4.1).

Localizing the ring of algebraic integers in $K$ by inverting a finite number of elements, we obtain a Dedekind ring $R \subset T$ and a Coxeter arrangement $A_{R}$ over $R$, such that $A_{T}$ is obtained from $A_{R}$ by extension of scalars. Let $\mathfrak{P}_{0} \in \operatorname{Spec} R$ be the intersection of the maximal ideal of $T$ with $R$. Then $\mathfrak{P}=\mathfrak{P}_{0}$ satisfies the conditions stated at the beginning of 4.2 .

Proposition 4.3.5. For $\mathfrak{P}=\mathfrak{P}_{0}$ or for $\mathfrak{P}$ any other maximal ideal of $R$ satisfying the conditions stated at the beginning of 4.2, the following holds:

(1) The representation of $\operatorname{Gal}(\bar{K} \mid K)$ on $\operatorname{det}\left(H_{c}\left(B_{T} \otimes \bar{K}, \delta^{*} \mathcal{L}_{\chi}\right)^{G}\right)^{-1}$ is unramified at $\mathfrak{P}$ and the action of the geometric Frobenius relative to $\mathfrak{P}$ is given by $-\varepsilon_{0}^{G}\left(B_{\mathfrak{P}} / k_{\mathfrak{P}}, \delta_{\mathfrak{P}}^{*} \mathcal{L}_{\chi_{\mathfrak{P}}}\right)$.

(2) The representation of $\operatorname{Gal}(\bar{K} \mid K)$ on $\operatorname{det}\left(H_{c}^{\cdot}\left(B_{0 T} \otimes \bar{K}, \delta^{*} \mathcal{L}_{\chi}\right)^{G}\right)^{-1}$ is unramified at $\mathfrak{P}$ and the action of the geometric Frobenius relative to $\mathfrak{P}$ is given by $\varepsilon_{0}^{G}\left(B_{0 \mathfrak{P}} / k_{\mathfrak{P}}, \delta_{\mathfrak{P}}^{*} \mathcal{L}_{\chi_{\mathfrak{P}}}\right)$. Moreover the Euler characteristic of $R \Gamma_{c}\left(B_{0 \mathfrak{P}} \otimes \bar{k}_{\mathfrak{P}}, \delta_{\mathfrak{P}}^{*} \mathcal{L}_{\chi \mathfrak{P}}\right)$ is zero.

Proof. The first statement in (1) follows directly from Lemma 4.3.1 and smooth base change. The second statement in (1) follows now from Corollary 4.3.2. For (2), observe that by Lemma 4.3.3 and the Leray spectral sequence the analogous statement holds for the Galois module $\operatorname{det}\left(H_{c}^{\cdot}\left(U_{T} \backslash B_{0 T} \otimes \bar{K}, \delta^{*} \mathcal{L}_{\chi}\right)^{G}\right)^{-1}$, by the same argument as at the end of the proof of Lemma 4.3.1, using the compactification $\mathbf{P}_{T}^{1}$ of $\mathbf{G}_{m, T}$. So it is enough to prove the analogue statement for $\operatorname{det}\left(H_{c}\left(U_{T} \otimes \bar{K}, \delta^{*} \mathcal{L}_{\chi}\right)^{G}\right)^{-1}$, which is clear if one considers the compactification used in the proof of Lemma 4.3.1. The assertion about the Euler characteristic follows in the same way.

4.4. Proof of the Main Theorem. Let $A=(V, G, q)$ be a Coxeter arrangement over $\mathbf{F}$. We assume that $p$ does not divide $|G|$. Let $d \geq 1$ be an integer and $\chi: \mathbf{F}^{\times} \rightarrow \overline{\mathbf{Q}}_{\ell}^{\times}$a character of order $d$. By Proposition 1.6, the Coxeter arrangement $A$ is liftable. We use the notation and material of 4.3. It follows from Proposition 1.3.3 that $\kappa \in T \backslash \mathfrak{P}_{0}$. It is well known (see [4] Ch.V, $\S 6$ Théorème 1 ) that $\sum_{i=1}^{n}\left(d_{i}-1\right)=N$. This implies that the right-hand side of 4.2.1 (1) may be expressed in terms of a Jacobi sum Hecke character ( $c f$. [26] Sommes trig. §6). By Proposition 4.3.5, Proposition 4.2.2 and Cebotarev density, we deduce that relations (1) and (2) in Proposition 4.2.2 are also valid for $\mathfrak{P}_{0}$. Together with Proposition 4.2.1 this gives

$$
\varepsilon_{0}^{G}\left(B / \mathbf{F}, \delta^{*} \mathcal{L}_{\chi}\right)=\varepsilon_{0}^{G}\left(B / \mathbf{F}, \overline{\mathbf{Q}}_{\ell}\right)
$$

$$
\cdot|\mathbf{F}|^{\sum_{\chi^{j} \neq 1} \beta_{j}} C \chi(\kappa)^{(-1)^{n}} \prod_{1 \leq i \leq n}\left[\frac{g\left((\phi \chi)^{d_{i}}\right)}{g(\phi \chi)}\right]^{(-1)^{n}} \frac{g\left(\chi^{N}\right)^{\bar{a}} g\left(\phi \chi^{N}\right)^{\bar{b}}}{g(\phi)^{\bar{b}}}
$$


and

$$
\varepsilon_{0}^{G}\left(B_{0} / \mathbf{F}, \delta^{*} \mathcal{L}_{\chi}\right)=\varepsilon_{0}^{G}\left(B_{0} / \mathbf{F}, \overline{\mathbf{Q}}_{\ell}\right)|\mathbf{F}|^{\sum_{\chi^{j} \neq 1} \beta_{j}} .
$$

Here we have implicitly used Proposition 1.5 to identify the degrees of $A$ and $A_{T}$.

By Lemma 4.3.3 the sheaves $R^{i} q_{T \mid U_{T} !} \delta_{T}^{*} \mathcal{L}_{\chi}$ are lisse on $\mathbf{G}_{m, T}$, hence we deduce from Proposition 3.2.1 (4) that $\left[\left(R q_{\mid U !} \delta^{*} \mathcal{L}_{\chi}\right)_{\bar{\eta}_{0}}^{G}\right]=\bar{a} V_{\chi^{N}}+\bar{b} V_{\phi \chi^{N}}$. The sheaves $\left(R^{i} q_{\mid U !} \delta^{*} \mathcal{L}_{\chi}\right)^{G}$ being lisse and tame on $\mathbf{G}_{m, \mathbf{F}}$ by Corollary 4.3 .4 , this implies, by the Leray spectral sequence, the relation

$$
\varepsilon_{0}^{G}\left(U \backslash B_{0} / \mathbf{F}, \delta^{*} \mathcal{L}_{\chi} \otimes q^{*} \mathcal{L}_{\psi}\right)=g\left(\chi^{N}\right)^{\bar{a}} g\left(\phi \chi^{N}\right)^{\bar{b}} \varepsilon_{0}^{G}\left(B / \mathbf{F}, \delta^{*} \mathcal{L}_{\chi}\right)^{-1} .
$$

(To verify this one uses the structure of tame irreducible lisse sheaves on $\mathbf{G}_{m, \mathbf{F}}$ (see, e.g., [16] p.198), or, alternatively, one can use [6] 8.1.4 and 3.1.4,(4) and [16] 3.1.5.5.) Since we have

$$
\begin{aligned}
\varepsilon_{0}^{G}\left(U / \mathbf{F}, \delta^{*} \mathcal{L}_{\chi}\right. & \left.\otimes q^{*} \mathcal{L}_{\psi}\right) \\
& =\varepsilon_{0}^{G}\left(B_{0} / \mathbf{F}, \delta^{*} \mathcal{L}_{\chi}\right) \varepsilon_{0}^{G}\left(U \backslash B_{0} / \mathbf{F}, \delta^{*} \mathcal{L}_{\chi} \otimes q^{*} \mathcal{L}_{\psi}\right),
\end{aligned}
$$

from equations (4.4.1) - (4.4.4), we obtain the relation

$$
\begin{aligned}
\varepsilon_{0}^{G}\left(U / \mathbf{F}, \delta^{*} \mathcal{L}_{\chi} \otimes q^{*} \mathcal{L}_{\psi}\right)=\frac{\varepsilon_{0}^{G}\left(B_{0} / \mathbf{F}, \overline{\mathbf{Q}}_{\ell}\right)}{\varepsilon_{0}^{G}\left(B / \mathbf{F}, \overline{\mathbf{Q}}_{\ell}\right)} \\
\cdot C^{-1} \chi(\kappa)^{(-1)^{n-1}} g(\phi)^{\bar{b}} \prod_{1 \leq i \leq n}\left[\frac{g\left((\phi \chi)^{d_{i}}\right)}{g(\phi \chi)}\right]^{(-1)^{n-1}} .
\end{aligned}
$$

So,

$$
\operatorname{dim}\left(-F, H_{c}^{\cdot}\left(U_{\overline{\mathbf{F}}}, \delta^{*} \mathcal{L}_{\chi} \otimes q^{*} \mathcal{L}_{\psi}\right)^{G}\right)=A\left[\chi(\kappa) \prod_{1 \leq i \leq n} \frac{g\left((\phi \chi)^{d_{i}}\right)}{g(\phi \chi)}\right]^{(-1)^{n}},
$$

with $A$ independent of the character $\chi$.

Now note that by Proposition 2.1.2 and Corollary 4.3.4, $H_{c}^{i}\left(U_{\overline{\mathbf{F}}}, \delta^{*} \mathcal{L}_{\chi} \otimes q^{*} \mathcal{L}_{\psi}\right)^{G}=$ 0 for $i \neq n$. On the other hand,

$$
\chi\left(R \Gamma_{c}\left(U_{\overline{\mathbf{F}}}, \delta^{*} \mathcal{L}_{\chi} \otimes q^{*} \mathcal{L}_{\psi}\right)^{G}\right)=\chi\left(R \Gamma_{c}\left(\mathbf{G}_{m, \overline{\mathbf{F}}},\left(R q_{\mid U !} \delta^{*} \mathcal{L}_{\chi}\right)^{G} \otimes \mathcal{L}_{\psi}\right)\right),
$$

because of Proposition 4.3.5 (2). Since the $\left(R^{i} q_{\mid U !} \delta^{*} \mathcal{L}_{\chi}\right)^{G}$ are lisse and tame on $\mathbf{G}_{m, \mathbf{F}}$, we obtain

$$
\chi\left(R \Gamma_{c}\left(\mathbf{G}_{m, \overline{\mathbf{F}}},\left(R q_{\mid U !} \delta^{*} \mathcal{L}_{\chi}\right)^{G} \otimes \mathcal{L}_{\psi}\right)\right)=-\sum_{i}(-1)^{i} \operatorname{dim} H_{c}^{i}\left(B \otimes \overline{\mathbf{F}}, \delta^{*} \mathcal{L}_{\chi}\right)^{G},
$$

see, e.g., [15] 4.8.2. By Corollary 4.3.2, we deduce that

$$
\operatorname{dim} H_{c}^{n}\left(U_{\overline{\mathbf{F}}}, \delta^{*} \mathcal{L}_{\chi} \otimes q^{*} \mathcal{L}_{\psi}\right)^{G}=1 .
$$

Hence we obtain by the Grothendieck trace formula,

$$
\begin{aligned}
S_{G}(\chi) & =\operatorname{Tr}\left(F, H_{c}\left(U_{\overline{\mathbf{F}}}, \delta^{*} \mathcal{L}_{\chi} \otimes q^{*} \mathcal{L}_{\psi}\right)^{G}\right), \\
& =A^{\prime} \chi(\kappa) \prod_{1 \leq i \leq n} \frac{g\left((\phi \chi)^{d_{i}}\right)}{g(\phi \chi)},
\end{aligned}
$$

with $A^{\prime}$ independent of the character $\chi$. To find the value of $A^{\prime}$ we take $\chi=\phi$, so $A^{\prime}=S_{G}(\phi) \phi(\kappa)$ and by Proposition 2.3.2 we obtain

$$
A^{\prime}=\phi(\kappa)(-1)^{n} \phi(\operatorname{discr} q) g(\phi)^{n} .
$$


The theorem now follows from (4.4.7) and (4.4.8).

4.5. Remark. A slightly different way of organizing the proof of the Main Theorem is by using material in [2] or [18] (or the results of [24]). This material provides an analogue of Theorem 4.1.1 for the determinant of period integrals. In this way Macdonald's formula (0.4), which yields the period $\int_{B(\mathbf{R})} \Delta^{s+1 / 2} \frac{d x}{d q}$, directly implies an analogous expression for $\varepsilon_{0}^{G}\left(B / \mathbf{F}, \delta^{*} \mathcal{L}_{\chi}\right) \varepsilon_{0}^{G}\left(B / \mathbf{F}, \overline{\mathbf{Q}}_{\ell}\right)^{-1}$, when $\chi$ is generic and $p \gg 0$, because the last expression and the period are given by similar formulas (compare Theorem 4.1.1 with loc. cit.). To obtain a full proof of the Main Theorem by this approach, one has to do similar calculations as in (4.4), but now one needs Proposition 3.2.1 (1) and Proposition 3.2.3.

4.6. Remark. That the analogy between our Main Theorem and Macdonald's formula $(0.4)$ is no coincidence, can be explained partially by the following conjecture of Deligne ([5] $\S 8.9)$ : the period of a rank one motive over a number field is completely determined by the Frobenius action. Using this conjecture one directly obtains from Macdonald's formula an expression for $\varepsilon_{0}^{G}\left(B / \mathbf{F}, \delta^{*} \mathcal{L}_{\chi}\right)$ when $\chi$ is generic and $p \gg 0$. Note that to apply the conjecture one first has to get rid of the additive character. (For this reduction one needs again Proposition 3.2.1 (1) and Proposition 3.2.3.)

\section{REFERENCES}

[1] G. Anderson. The evaluation of Selberg sums. C.R. Acad. Sci. Paris, 311:469-472, 1990. MR 91m:11109

[2] G. Anderson. Local factorisation of twisted DR cohomology groups. Compositio Math., 83:69105, 1992. MR 93k: 14031

[3] V. Arnold, A. Varchenko, and S. Goussein-Zadé. Singularités des applications différentiables. Vol. 2 (Monodromie et comportement asymptotique des intégrales), Editions Mir, Moscou, 1986.

[4] N. Bourbaki. Groupes et algèbres de Lie. Chapitres 4, 5 et 6, Hermann, 1968. MR 39:1590

[5] P. Deligne. Valeurs de fonctions $L$ et périodes d'intégrales. In A. Borel and W. Casselman, editors, Automorphic forms, Representations and L-functions, Proceedings of Symposia in Pure Mathematics, pages 313-346, 1979. MR 81d:12009

[6] J. Denef and A. Gyoja. Character sums associated to prehomogeneous vector spaces. Compositio Math., to appear.

[7] J. Denef and F. Loeser. Macdonald integrals and monodromy. In preparation.

[8] J. Denef and F. Loeser. Weights of exponential sums, intersection cohomology, and Newton polyhedra. Invent. Math., 106:275-294, 1991. MR 93a:14019

[9] J. Denef and F. Loeser. Détermination géométrique des sommes de Selberg-Evans. Bull. Soc. math. France, 122:533-551, 1994. MR 96f:14022

[10] J. Denef and F. Loeser. Regular elements and monodromy of discriminants of finite reflection groups. Indag. Math., 6:129-143, 1995. MR 96h:20077

[11] R. J. Evans. Identities for products of Gauss sums over finite fields. L'Enseignement Mathématique, 27:197-209, 1981. MR 83i:10050

[12] R. J. Evans. Character sums over finite fields. Lecture Notes in Pure and Applied Mathematics, 141:57-73, 1991. MR 94c:11118

[13] R. J. Evans. The evaluation of Selberg character sums. L'Enseignement Mathématique, 37:235-248, 1991. MR 93c:11062

[14] M. Greenberg Rational points in henselian discrete valuation rings. Publ. Math. I.H.E.S., 31:59-64, 1966. MR 34:7515

[15] N. Katz. Sommes exponentielles. Astérisque, 79, 1980. MR 82m:10059

[16] G. Laumon. Transformation de Fourier, constantes d'équations fonctionnelles et conjecture de Weil. Publ. Math. I.H.E.S., 65:131-210, 1987. MR 88g:14019

[17] F. Loeser. Arrangements d'hyperplans et sommes de Gauss. Ann. scient. Éc. Norm. Sup., 24:379-400, 1991. MR 93c:11063 
[18] F. Loeser and C. Sabbah. Equations aux différences finies et déterminants de fonctions multiformes. Comment. Math. Helvetici, 66:458-503, 1991. MR 93a:32057

[19] I. G. Macdonald. Some conjectures for root systems. SIAM J. Math. An., 13:988-1007, 1982. MR 84h:17006a

[20] J. S. Milne. Étale Cohomology. Princeton University Press, 1980. MR 81j:14002

[21] E. M. Opdam. Some applications of hypergeometric shift operators. Invent. Math., 98:1-18, 1989. MR 91h:33024

[22] P. Orlik and H. Terao. Arrangements of hyperplanes. Springer-Verlag, Berlin Heidelberg, 1992. MR 94e:52014

[23] T. Saito. Jacobi sum Hecke characters, de Rham discriminant, and the determinant of $\ell$-adic cohomologies. Journal of Algebraic Geometry, 3:411-434, 1994. MR 95f:14036

[24] T. Saito and T. Terasoma. Determinant of period integrals. J. Amer. Math. Soc. 10 (1997), 865-937. MR 98c: 14004

[25] J.-P. Serre. Représentations linéaires des groupes finis. Hermann, Paris, 1967, deuxième édition, refondue, 1971. MR 50:4718

[26] Séminaire de Géométrie Algébrique du Bois-Marie, SGA $4 \frac{1}{2}$, Cohomologie étale, par P. Deligne, avec la collaboration de J.-F. Boutot, A. Grothendieck, L. Illusie et J.-L. Verdier, Lecture Notes in Math., vol. 569, Springer-Verlag 1977. MR 57:3132

[27] Séminaire de Géométrie Algébrique du Bois-Marie, SGA 7, Groupes de monodromie en géométrie algébrique, dirigé par A. Grothendieck avec la collaboration de M. Raynaud et D. S. Rim pour la partie I et par P. Deligne et N. Katz pour la partie II, Lecture Notes in Math., vol. 288 et 340, Springer-Verlag 1972 et 1973. MR 50:7134

Department of Mathematics, University of Leuven, Celestijnenlaan 200B, 3001 LeuVEN, BELGIUM

E-mail address: Jan.Denef@wis.kuleuven.ac.be

Centre de Mathématiques, Ecole Polytechnique, F-91128 Palaiseau (URA 169 du CNRS), and Institut de Mathématiques, Université P. et M. Curie, Case 82, 4 place Jussieu, F-75252 Paris Cedex 05 (UMR 9994 du CNRS)

E-mail address: loeser@math.polytechnique.fr 